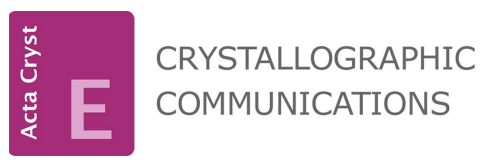

ISSN 2056-9890

Received 12 September 2015

Accepted 16 September 2015

Edited by A. J. Lough, University of Toronto, Canada

Keywords: crystal structure; bromanilic acid; morpholine; hydrogen-bonding; proton disorder

CCDC references: $1424714 ; 1424713$; 1424712

Supporting information: this article has supporting information at journals.iucr.org/e

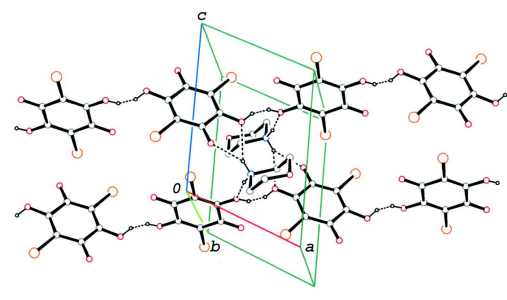

OPEN $\odot$ ACCESS

\section{Crystal structures of morpholinium hydrogen bromanilate at 130, 145 and $180 \mathrm{~K}$}

\author{
Kazuma Gotoh, Yuki Tahara and Hiroyuki Ishida*
}

Department of Chemistry, Faculty of Science, Okayama University, Okayama 700-8530, Japan. *Correspondence e-mail: ishidah@cc.okayama-u.ac.jp

Crystal structures of the title compound (systematic name: morpholin-4-ium 2,5-dibromo-4-hydroxy-3,6-dioxocyclohexa-1,4-dien-1-olate), $\quad \mathrm{C}_{4} \mathrm{H}_{10} \mathrm{NO}^{+}$.$\mathrm{C}_{6} \mathrm{HBr}_{2} \mathrm{O}_{4}{ }^{-}$, were determined at three temperatures, viz. 130, 145 and $180 \mathrm{~K}$. The asymmetric unit comprises one morpholinium cation and two halves of crystallographically independent bromanilate monoanions, which are located on inversion centres. The conformations of the two independent bromanilate anions are different from each other with respect to the $\mathrm{O}-\mathrm{H}$ orientation. In the crystal, the two different anions are linked alternately into a chain along [211] through a short $\mathrm{O}-\mathrm{H} \cdots \mathrm{O}$ hydrogen bond, in which the $\mathrm{H}$ atom is disordered over two positions. The refined site-occupancy ratios, which are almost constant in the temperature range studied, are $0.49(3): 0.51$ (3), 0.52 (3):0.48 (3) and 0.50 (3):0.50 (3), respectively, at 130, 145 and $180 \mathrm{~K}$, and no significant difference in the molecular geometry and the molecular packing is observed at the three temperatures. The morpholinium cation links adjacent chains of anions via $\mathrm{N}-\mathrm{H} \cdots \mathrm{O}$ hydrogen bonds, forming a sheet structure parallel to (111).

\section{Chemical context}

Anilic acid (2,5-dihydroxy-1,4-benzoquinone) derivatives, such as chloranilic acid (2,5-dichloro-3,6-dihydroxy-1,4benzoqinone) and bromanilic acid (2,5-dibromo-3,6-dihydroxy-1,4-benzoqinone), appear particularly attractive as a versatile template for generating hydrogen-bonded selfassemblies with various organic bases (Zaman et al., 2001; Molčanov \& Kojić-Prodić, 2010; Gotoh \& Ishida, 2011; Thomas et al., 2013) and also as a model compound for investigating proton dynamics in hydrogen-bond systems (Ikeda et al., 2005; Seliger et al., 2009). Furthermore, salts and co-crystals of anilic acids with organic bases have attracted much interest with respect to organic ferroelectrics (Horiuchi et al., 2008, 2009, 2013).

In our previous study, we reported the crystal structure of morpholinium hydrogen chloranilate, $\mathrm{C}_{4} \mathrm{H}_{10} \mathrm{NO}^{+} \cdot \mathrm{C}_{6} \mathrm{HCl}_{2} \mathrm{O}_{4}{ }^{-}$, in which a short $\mathrm{O}-\mathrm{H} \cdots \mathrm{O}$ hydrogen bond is formed between the chloranilate ions and the $\mathrm{H}$ atom in the hydrogen bond is disordered over two sites (Ishida \& Kashino, 1999). The measurements of ${ }^{35} \mathrm{Cl} \mathrm{NQR}$ (nuclear quadrupole resonance) for the compound in the temperature range 4-300 K showed an anomalous temperature dependence of the NQR frequencies, which cannot be explained by the conventional Bayer-type lattice motion: one of the two frequencies exhibits an anomalous increase with increasing temperature from $4.2 \mathrm{~K}$ while the other frequency shows a rather fast decrease with temperature. The anomalous behavior was ascribed to a drastic temperature variation of the disordered $\mathrm{O}-\mathrm{H} \cdots \mathrm{O}$ 
hydrogen bond, as revealed by multi-temperature X-ray diffraction (Tobu et al., 2012). In the present study, we have undertaken the structural determination of morpholinium hydrogen bromanilate, $\mathrm{C}_{4} \mathrm{H}_{10} \mathrm{NO}^{+} \cdot \mathrm{C}_{6} \mathrm{HBr}_{2} \mathrm{O}_{4}{ }^{-}$, to extend the study of hydrogen-bonding in the amine-halohydroxybenzoquinone system.

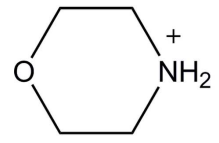<smiles>O=C1C(=O)C(Br)C(=O)C(O)=C1Br</smiles>

\section{Structural commentary}

The title compound is isomorphous with morpholinium hydrogen chloranilate in the space group $P \overline{1}$ (Ishida \& Kashino, 1999; Tobu et al., 2012) and has a quite similar molecular packing to the chloranilate. The asymmetric unit of the title compound comprises one morpholinium cation and two halves of crystallographically independent bromanilate monoanions, which are each located on an inversion centre (Fig. 1). The conformations of two bromanilate anions are different from each other with respect to the $\mathrm{O}-\mathrm{H}$ orientation as shown schematically in Fig. 2.

In morpholinium hydrogen chloranilate, the bond distances of $\mathrm{C} 3-\mathrm{O} 2$ and $\mathrm{C} 6-\mathrm{O} 4$, which are involved in the disordered $\mathrm{O}-\mathrm{H} \cdots \mathrm{O}$ hydrogen bond, showed slight but systematic decrease and increase, respectively, with temperature [C3O2: from $1.2994(10) \AA$ at $114 \mathrm{~K}$ to $1.2951(10) \AA$ at $180 \mathrm{~K}$;

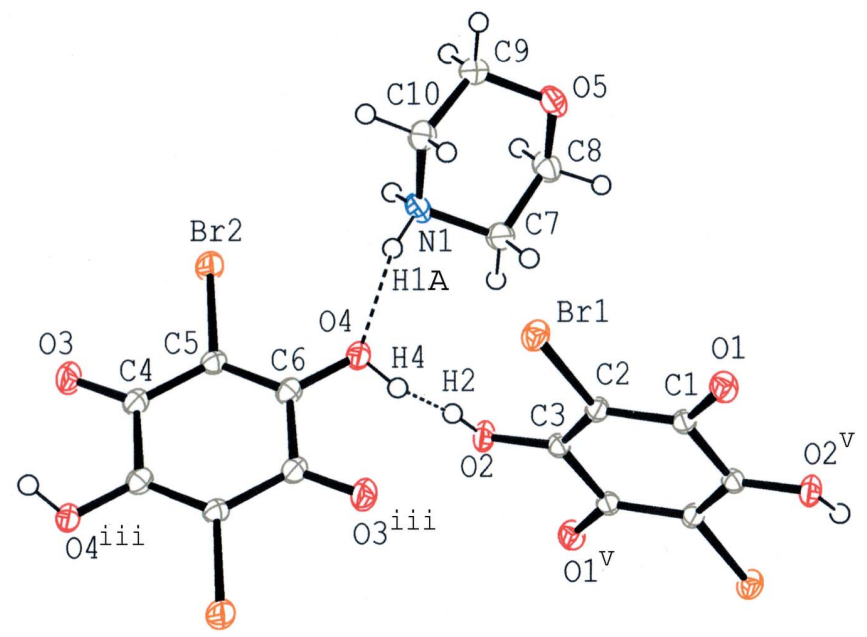

Figure 1

A view of the molecular structure of the title compound at $180 \mathrm{~K}$, showing the atom-numbering scheme. Displacement ellipsoids of non-H atoms are drawn at the $50 \%$ probability level and $\mathrm{H}$ atoms are drawn as circles of arbitrary size. The site-occupancy factors of the disordered $\mathrm{H}$ atom $(\mathrm{H} 2$ and $\mathrm{H} 4)$ are approximately equal. The $\mathrm{N}-\mathrm{H} \cdots \mathrm{O}$ and $\mathrm{O}-\mathrm{H} \cdots \mathrm{O}$ hydrogen bonds are indicated by dashed lines. [Symmetry codes: (iii) $-x$, $-y+1,-z ;(\mathrm{v})-x+2,-y+2,-z+1$.]
Table 1

Hydrogen-bond geometry $\left(\AA,^{\circ}\right)$ at $130 \mathrm{~K}$.

\begin{tabular}{lllll}
\hline$D-\mathrm{H} \cdots A$ & $D-\mathrm{H}$ & $\mathrm{H} \cdots A$ & $D \cdots A$ & $D-\mathrm{H} \cdots A$ \\
\hline $\mathrm{N} 1-\mathrm{H} 1 A \cdots \mathrm{O} 4$ & $0.88(3)$ & $2.03(3)$ & $2.886(2)$ & $166(2)$ \\
$\mathrm{N} 1-\mathrm{H} 1 B \cdots 1^{\mathrm{i}}$ & $0.86(3)$ & $2.16(3)$ & $2.938(2)$ & $150(2)$ \\
$\mathrm{N} 1-\mathrm{H} 1 B \cdots \mathrm{O} 2^{\mathrm{ii}}$ & $0.86(3)$ & $2.27(3)$ & $2.955(2)$ & $137(2)$ \\
$\mathrm{O} 2-\mathrm{H} 2 \cdots \mathrm{O} 4$ & $0.81(3)$ & $1.77(3)$ & $2.5160(16)$ & $152(4)$ \\
$\mathrm{O} 2-\mathrm{H} 2 \cdots \mathrm{O}{ }^{\text {iii }}$ & $0.81(3)$ & $2.57(3)$ & $3.0613(17)$ & $120(3)$ \\
$\mathrm{O} 4-\mathrm{H} 4 \cdots \mathrm{O} 2$ & $0.82(3)$ & $1.82(4)$ & $2.5160(16)$ & $143(4)$ \\
$\mathrm{C} 7-\mathrm{H} 7 A \cdots 4^{\mathrm{ii}}$ & 0.99 & 2.53 & $3.391(2)$ & 145 \\
$\mathrm{C} 10-\mathrm{H} 10 B \cdots \mathrm{Br}^{\mathrm{iv}}$ & 0.99 & 2.90 & $3.8892(17)$ & 175
\end{tabular}

Symmetry codes: (i) $x-1, y-1, z$; (ii) $-x+1,-y+1,-z+1$; (iii) $-x,-y+1,-z$; (iv) $-x,-y,-z$.

Table 2

Hydrogen-bond geometry $\left(\AA{ }^{\circ}\right)$ at $145 \mathrm{~K}$.

\begin{tabular}{lllll}
\hline$D-\mathrm{H} \cdots A$ & $D-\mathrm{H}$ & $\mathrm{H} \cdots A$ & $D \cdots A$ & $D-\mathrm{H} \cdots A$ \\
\hline $\mathrm{N} 1-\mathrm{H} 1 A \cdots \mathrm{O} 4$ & $0.86(3)$ & $2.04(3)$ & $2.888(2)$ & $166(2)$ \\
$\mathrm{N} 1-\mathrm{H} 1 B \cdots \mathrm{O} 1^{\mathrm{i}}$ & $0.85(3)$ & $2.17(3)$ & $2.938(2)$ & $151(2)$ \\
$\mathrm{N} 1-\mathrm{H} 1 B \cdots \mathrm{O} 2^{\mathrm{ii}}$ & $0.85(3)$ & $2.29(3)$ & $2.959(2)$ & $136(2)$ \\
$\mathrm{O} 2-\mathrm{H} 2 \cdots \mathrm{O} 4$ & $0.82(3)$ & $1.77(3)$ & $2.5174(16)$ & $153(4)$ \\
$\mathrm{O} 2-\mathrm{H} 2 \cdots \mathrm{O}{ }^{\text {iii }}$ & $0.82(3)$ & $2.58(3)$ & $3.0628(17)$ & $120(3)$ \\
$\mathrm{O} 4-\mathrm{H} 4 \cdots \mathrm{O} 2$ & $0.82(3)$ & $1.79(4)$ & $2.5174(16)$ & $147(4)$ \\
$\mathrm{C} 7-\mathrm{H} 7 A \cdots \mathrm{O} 4^{\mathrm{ii}}$ & 0.99 & 2.54 & $3.394(2)$ & 145 \\
$\mathrm{C} 10-\mathrm{H} 10 B \cdots \mathrm{Br} 2^{\text {iv }}$ & 0.99 & 2.90 & $3.8905(17)$ & 175
\end{tabular}

Symmetry codes: (i) $x-1, y-1, z$; (ii) $-x+1,-y+1,-z+1$; (iii) $-x,-y+1,-z$; (iv) $-x,-y,-z$.

Table 3

Hydrogen-bond geometry $\left(\AA{ }^{\circ}\right)$ at $180 \mathrm{~K}$.

\begin{tabular}{lllll}
\hline$D-\mathrm{H} \cdots A$ & $D-\mathrm{H}$ & $\mathrm{H} \cdots A$ & $D \cdots A$ & $D-\mathrm{H} \cdots A$ \\
\hline $\mathrm{N} 1-\mathrm{H} 1 A \cdots \mathrm{O} 4$ & $0.89(3)$ & $2.02(3)$ & $2.890(2)$ & $167(2)$ \\
$\mathrm{N} 1-\mathrm{H} 1 B \cdots 1^{\mathrm{i}}$ & $0.86(3)$ & $2.16(3)$ & $2.938(2)$ & $150(2)$ \\
$\mathrm{N} 1-\mathrm{H} 1 B \cdots \mathrm{O} 2^{\mathrm{ii}}$ & $0.86(3)$ & $2.28(3)$ & $2.964(2)$ & $136(2)$ \\
$\mathrm{O} 2-\mathrm{H} 2 \cdots \mathrm{O} 4$ & $0.82(3)$ & $1.79(4)$ & $2.5224(16)$ & $148(5)$ \\
$\mathrm{O} 2-\mathrm{H} 2 \cdots \mathrm{O}{ }^{\mathrm{iii}}$ & $0.82(3)$ & $2.55(4)$ & $3.0678(18)$ & $122(4)$ \\
$\mathrm{O} 4-\mathrm{H} 4 \cdots \mathrm{O} 2$ & $0.82(3)$ & $1.80(4)$ & $2.5224(16)$ & $147(4)$ \\
$\mathrm{C} 7-\mathrm{H} 7 A \cdots 4^{\mathrm{ii}}$ & 0.99 & 2.55 & $3.402(2)$ & 145 \\
$\mathrm{C} 10-\mathrm{H} 10 B \cdots \mathrm{Br} 2^{\text {iv }}$ & 0.99 & 2.91 & $3.8946(17)$ & 174
\end{tabular}

Symmetry codes: (i) $x-1, y-1, z$; (ii) $-x+1,-y+1,-z+1$; (iii) $-x,-y+1,-z$; (iv) $-x,-y,-z$.

C6-O4: from 1.290 (10) $\AA$ at $114 \mathrm{~K}$ to 1.2946 (10) at $180 \mathrm{~K}]$, which corresponds to population changes of the two disordered proton sites in the hydrogen bond (Tobu et al., 2012). In the present compound, however, the $\mathrm{C} 3-\mathrm{O} 2$ and $\mathrm{C} 6-\mathrm{O} 4$ bond lengths are almost constant [C3-O2: $1.2953(17)$,<smiles>O=c1c(O)c(Br)c(=O)c(=O)c1=O</smiles>

$\boldsymbol{A}$<smiles>O=C1C(O)=C(O)C(=O)C(Br)=C1O</smiles>

$\boldsymbol{B}$
Figure 2

Two conformations ( $A$ and $B$ forms) of bromanilic acid with respect to the $\mathrm{O}-\mathrm{H}$ orientation. 


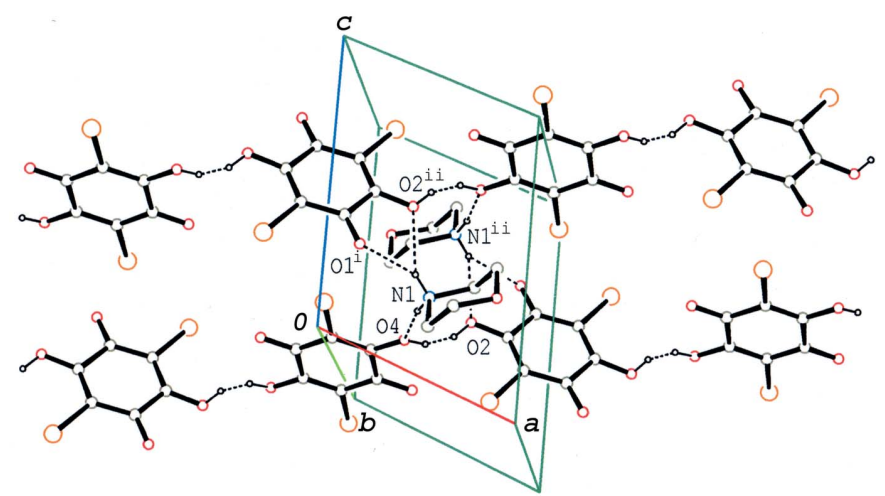

Figure 3

A partial packing diagram of the title compound at $180 \mathrm{~K}$, showing the hydrogen-bonded aggregate of morpholinium and hydrogen bromanilate ions. The $\mathrm{N}-\mathrm{H} \cdots \mathrm{O}$ and $\mathrm{O}-\mathrm{H} \cdots \mathrm{O}$ hydrogen bonds are indicated by dashed lines. [Symmetry codes: (i) $x-1, y-1, z$; (ii) $-x+1,-y+1$, $-z+1$.]

1.2937 (17) and 1.2931 (17) $\AA$ at 130, 145 and $180 \mathrm{~K}$; C6-O4: 1.3002 (18), $1.2997(18)$ and $1.2997(18) \AA$ at 130, 145 and $180 \mathrm{~K}$ ] and no significant difference in the molecular geometry is observed at the three temperatures.

\section{Supramolecular features}

In the crystal, the two independent bromanilate anions with different conformations are linked alternately by short $\mathrm{O}-$ $\mathrm{H}$... O hydrogen bonds (Tables 1, 2 and 3), forming a chain along [211] (Fig. 3). The adjacent independent anions are almost perpendicular to each other, with dihedral angles of $86.57(7)^{\circ}(130 \mathrm{~K}), 86.65(7)^{\circ}(145 \mathrm{~K})$ and $86.81(7)^{\circ}(180 \mathrm{~K})$ between the benzoquinone rings. The morpholinium cation connects the anion chains through $\mathrm{N}-\mathrm{H}$. . O hydrogen bonds and a weak $\mathrm{C}-\mathrm{H} \cdots \mathrm{O}$ hydrogen bond into a sheet parallel to (111) (Fig. 4). Between the chains, short $\mathrm{Br} \cdots \mathrm{O}$ and $\mathrm{Br} \cdots \mathrm{C}$

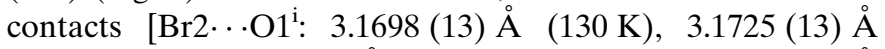
$(145 \mathrm{~K})$ and $3.1763(13) \AA(180 \mathrm{~K})$; Br2 $\cdots \mathrm{C}^{\mathrm{i}}$ : $3.2673(15) \AA$ $(130 \mathrm{~K}), 3.2716(15) \AA(145 \mathrm{~K})$ and $3.2808(15) \AA(180 \mathrm{~K})$; symmetry code: (i) $x$ - $1, y-1, z]$ are observed. A weak $\mathrm{C}-$ $\mathrm{H} \cdots \mathrm{Br}$ interaction is also observed between the sheets.

\section{Database survey}

Although a search of the Cambridge Structural Database (Version 5.36, last update February 2015; Groom \& Allen, 2014) for organic salts and co-crystals with bromanilic acid gave 31 hits, no crystal structure including the $A$ form (Fig. 2) was found.

\section{Synthesis and crystallization}

Single crystals of the title compound suitable for X-ray diffraction were prepared by slow evaporation from an acetonitrile solution $(200 \mathrm{ml})$ of bromanilic acid $(200 \mathrm{mg})$ with morpholine $(60 \mathrm{mg})$ at room temperature.

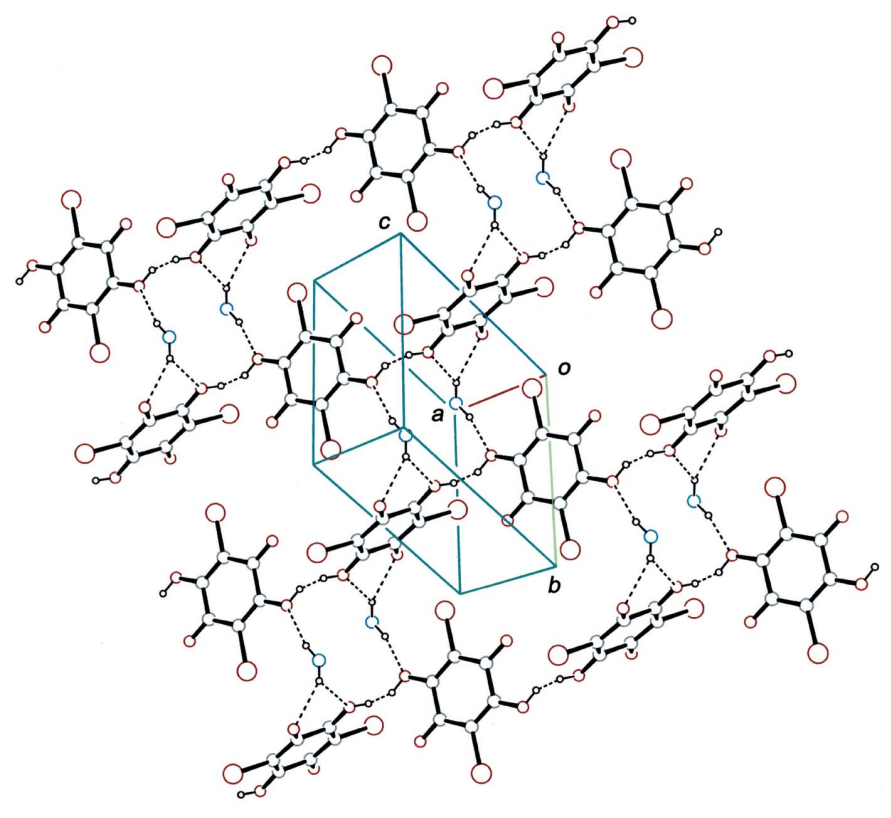

Figure 4

A packing diagram of the title compound at $180 \mathrm{~K}$, showing the sheet structure formed through $\mathrm{N}-\mathrm{H} \cdots \mathrm{O}$ and $\mathrm{O}-\mathrm{H} \cdots \mathrm{O}$ hydrogen bonds (dashed lines). For the morpholinium cations, only $\mathrm{NH}_{2}$ groups are shown for clarity.

\section{Refinement}

Crystal data, data collection and structure refinement details are summarized in Table 4. C-bound $\mathrm{H}$ atoms of the morpholinium cation were positioned geometrically with $\mathrm{C}-$ $\mathrm{H}=0.99 \AA$ and were refined as riding with $U_{\text {iso }}(\mathrm{H})=$ $1.2 U_{\text {eq }}(\mathrm{C})$. The $\mathrm{N}$-bound $\mathrm{H}$ atom was located in a difference Fourier map and refined freely [refined $\mathrm{N}-\mathrm{H}=0.85$ (3)0.89 (3) $\AA$ ] . Two disordered positions of the $\mathrm{H}$ atom in the $\mathrm{O}-$ $\mathrm{H}$... O hydrogen bond were located in a difference Fourier map. Since site occupancy factors and isotropic displacement parameters are correlated and bonding effects also make the site-occupancy factors less reliable, the positional parameters and the occupancies of the $\mathrm{H}$ atom were refined, with $U_{\text {iso }}(\mathrm{H})$ $=1.5 U_{\text {eg }}(\mathrm{O})$, and with distance restraints of $\mathrm{O}-\mathrm{H}=$ 0.84 (2) А.

\section{References}

Altomare, A., Cascarano, G., Giacovazzo, C., Guagliardi, A., Burla, M., Polidori, G. \& Camalli, M. (1994). J. Appl. Cryst. 27, 435.

Farrugia, L. J. (2012). J. Appl. Cryst. 45, 849-854.

Gotoh, K. \& Ishida, H. (2011). Acta Cryst. C67, o500-0504.

Groom, C. R. \& Allen, F. H. (2014). Angew. Chem. Int. Ed. 53, 662671.

Higashi, T. (1999). NUMABS. Rigaku Corporation, Tokyo, Japan.

Horiuchi, S., Kumai, R., Tokunaga, Y. \& Tokura, Y. (2008). J. Am. Chem. Soc. 130, 13382-13391.

Horiuchi, S., Kumai, R. \& Tokura, Y. (2009). J. Mater. Chem. 19, $4421-4434$.

Horiuchi, S., Kumai, R. \& Tokura, Y. (2013). J. Am. Chem. Soc. 135, 4492-4500.

Ikeda, R., Takahashi, S., Nihei, T., Ishihara, H. \& Ishida, H. (2005). Bull. Chem. Soc. Jpn, 78, 1241-1245.

Ishida, H. \& Kashino, S. (1999). Acta Cryst. C55, 1923-1926. 
Table 4

Experimental details.

\begin{tabular}{|c|c|c|c|}
\hline & $130 \mathrm{~K}$ & $145 \mathrm{~K}$ & $180 \mathrm{~K}$ \\
\hline \multicolumn{4}{|l|}{ Crystal data } \\
\hline Chemical formula & $\mathrm{C}_{4} \mathrm{H}_{10} \mathrm{NO}^{+} \cdot \mathrm{C}_{6} \mathrm{HBr}_{2} \mathrm{O}_{4}^{-}$ & $\mathrm{C}_{4} \mathrm{H}_{10} \mathrm{NO}^{+} \cdot \mathrm{C}_{6} \mathrm{HBr}_{2} \mathrm{O}_{4}^{-}$ & $\mathrm{C}_{4} \mathrm{H}_{10} \mathrm{NO}^{+} \cdot \mathrm{C}_{6} \mathrm{HBr}_{2} \mathrm{O}_{4}^{-}$ \\
\hline$M_{\mathrm{r}}$ & 385.01 & 385.01 & 385.01 \\
\hline$a, b, c(\AA)$ & $8.62046(19), 9.2129(2), 9.4257$ (2) & $\begin{array}{l}8.62293(18), 9.21849(19) \\
\quad 9.4354(2)\end{array}$ & $\begin{array}{l}8.62824(17), 9.23087(18) \\
\quad 9.46007(19)\end{array}$ \\
\hline$\alpha, \beta, \gamma\left({ }^{\circ}\right)$ & $\begin{array}{l}93.5208(7), 112.9139(7) \\
\quad 115.9757(7)\end{array}$ & $\begin{array}{l}93.5239(7), 112.9190(7) \\
115.9777(7)\end{array}$ & $\begin{array}{l}93.5321(7), 112.9738(7) \\
\quad 115.9508(7)\end{array}$ \\
\hline Radiation type & Mo $K \alpha$ & Mo $K \alpha$ & Mo $K \alpha$ \\
\hline$\mu\left(\mathrm{mm}^{-1}\right)$ & 6.84 & 6.83 & 6.80 \\
\hline Crystal size (mm) & $0.40 \times 0.34 \times 0.18$ & $0.40 \times 0.34 \times 0.18$ & $0.40 \times 0.34 \times 0.18$ \\
\hline \multicolumn{4}{|l|}{ Data collection } \\
\hline Diffractometer & Rigaku R-AXIS RAPIDII & Rigaku R-AXIS RAPIDII & Rigaku R-AXIS RAPIDII \\
\hline Absorption correction & $\begin{array}{l}\text { Numerical (NUMABS; Higashi, } \\
\text { 1999) }\end{array}$ & $\begin{array}{l}\text { Numerical (NUMABS; Higashi, } \\
\text { 1999) }\end{array}$ & $\begin{array}{l}\text { Numerical (NUMABS; Higashi, } \\
\text { 1999) }\end{array}$ \\
\hline$(\sin \theta / \lambda)_{\max }\left(\AA^{-1}\right)$ & 0.704 & 0.704 & 0.703 \\
\hline \multicolumn{4}{|l|}{ Refinement } \\
\hline$R\left[F^{2}>2 \sigma\left(F^{2}\right)\right], w R\left(F^{2}\right), S$ & $0.017,0.046,1.14$ & $0.018,0.046,1.10$ & $0.019,0.048,1.09$ \\
\hline No. of reflections & 3468 & 3473 & 3487 \\
\hline No. of parameters & 178 & 178 & 178 \\
\hline No. of restraints & 2 & 2 & 2 \\
\hline $\mathrm{H}$-atom treatment & $\begin{array}{l}\mathrm{H} \text { atoms treated by a mixture of } \\
\text { independent and constrained } \\
\text { refinement }\end{array}$ & $\begin{array}{l}\mathrm{H} \text { atoms treated by a mixture of } \\
\text { independent and constrained } \\
\text { refinement }\end{array}$ & $\begin{array}{l}\mathrm{H} \text { atoms treated by a mixture of } \\
\text { independent and constrained } \\
\text { refinement }\end{array}$ \\
\hline$\Delta \rho_{\max }, \Delta \rho_{\min }\left(\mathrm{e} \AA^{-3}\right)$ & $0.50,-0.37$ & $0.48,-0.44$ & $0.59,-0.45$ \\
\hline
\end{tabular}

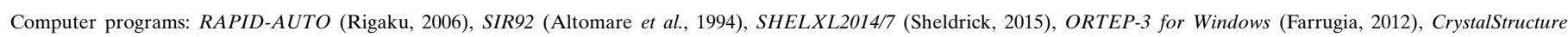
(Rigaku, 2010) and PLATON (Spek, 2009).

Molčanov, K. \& Kojić-Prodić, B. (2010). CrystEngComm, 12, 925-939. Rigaku (2006). RAPID-AUTO. Rigaku Corporation, Tokyo, Japan. Rigaku (2010). CrystalStructure. Rigaku Corporation, Tokyo, Japan.

Seliger, J., Žagar, V., Gotoh, K., Ishida, H., Konnai, A., Amino, D. \& Asaji, T. (2009). Phys. Chem. Chem. Phys. 11, 2281-2286.

Sheldrick, G. M. (2015). Acta Cryst. C71, 3-8.
Spek, A. L. (2009). Acta Cryst. D65, 148-155.

Thomas, L. H., Adam, M. S., O'Neill, A. \& Wilson, C. C. (2013). Acta Cryst. C69, 1279-1288.

Tobu, Y., Ikeda, R., Nihei, T., Gotoh, K., Ishida, H. \& Asaji, T. (2012). Phys. Chem. Chem. Phys. 14, 12347-12354.

Zaman, Md. B., Tomura, M. \& Yamashita, Y. (2001). J. Org. Chem. 66, 5987-5995. 


\section{supporting information}

Acta Cryst. (2015). E71, 1226-1229 [doi:10.1107/S2056989015017272]

\section{Crystal structures of morpholinium hydrogen bromanilate at 130, 145 and 180 K}

\section{Kazuma Gotoh, Yuki Tahara and Hiroyuki Ishida}

\section{Computing details}

For all compounds, data collection: RAPID-AUTO (Rigaku, 2006); cell refinement: RAPID-AUTO (Rigaku, 2006); data reduction: RAPID-AUTO (Rigaku, 2006); program(s) used to solve structure: SIR92 (Altomare et al., 1994). Program(s) used to refine structure: SHELXL2014 (Sheldrick, 2015) for (1); SHELXL2014/7 (Sheldrick, 2015) for (2), (3). For all compounds, molecular graphics: ORTEP-3 for Windows (Farrugia, 2012); software used to prepare material for publication: CrystalStructure (Rigaku, 2010) and PLATON (Spek, 2009).

(1) Morpholin-4-ium 2,5-dibromo-4-hydroxy-3,6-dioxocyclohexa-1,4-dien-1-olate

Crystal data

$\mathrm{C}_{4} \mathrm{H}_{10} \mathrm{NO}^{+} \cdot \mathrm{C}_{6} \mathrm{HBr}_{2} \mathrm{O}_{4}^{-}$

$M_{r}=385.01$

Triclinic, $P \overline{1}$

Hall symbol: -P 1

$a=8.62046(19) \AA$

$b=9.2129(2) \AA$

$c=9.4257(2) \AA$

$\alpha=93.5208(7)^{\circ}$

$\beta=112.9139(7)^{\circ}$

$\gamma=115.9757(7)^{\circ}$

$V=595.05(3) \AA^{3}$

Data collection

Rigaku R-AXIS RAPIDII

diffractometer

Detector resolution: 10.000 pixels $\mathrm{mm}^{-1}$

$\omega$ scans

Absorption correction: numerical

(NUMABS; Higashi, 1999)

$T_{\min }=0.096, T_{\max }=0.292$

18162 measured reflections

Refinement

Refinement on $F^{2}$

Least-squares matrix: full

$R\left[F^{2}>2 \sigma\left(F^{2}\right)\right]=0.017$

$w R\left(F^{2}\right)=0.046$

$S=1.14$

3468 reflections
$Z=2$

$F(000)=376.00$

$D_{\mathrm{x}}=2.149 \mathrm{Mg} \mathrm{m}^{-3}$

Mo $K \alpha$ radiation, $\lambda=0.71075 \AA$

Cell parameters from 15996 reflections

$\theta=3.0-30.1^{\circ}$

$\mu=6.84 \mathrm{~mm}^{-1}$

$T=130 \mathrm{~K}$

Block, brown

$0.40 \times 0.34 \times 0.18 \mathrm{~mm}$

3468 independent reflections

3183 reflections with $I>2 \sigma(I)$

$R_{\text {int }}=0.026$

$\theta_{\text {max }}=30.0^{\circ}, \theta_{\min }=3.0^{\circ}$

$h=-12 \rightarrow 12$

$k=-12 \rightarrow 12$

$l=-13 \rightarrow 13$

178 parameters

2 restraints

Hydrogen site location: mixed

$\mathrm{H}$ atoms treated by a mixture of independent and constrained refinement 
$w=1 /\left[\sigma^{2}\left(F_{\mathrm{o}}^{2}\right)+(0.0195 P)^{2}+0.3723 P\right]$

where $P=\left(F_{\mathrm{o}}^{2}+2 F_{\mathrm{c}}^{2}\right) / 3$

$(\Delta / \sigma)_{\max }=0.002$

$$
\Delta \rho_{\max }=0.50 \text { e } \AA^{-3}
$$$$
\Delta \rho_{\min }=-0.37 \text { e } \AA^{-3}
$$

\section{Special details}

Geometry. All e.s.d.'s (except the e.s.d. in the dihedral angle between two 1.s. planes) are estimated using the full covariance matrix. The cell e.s.d.'s are taken into account individually in the estimation of e.s.d.'s in distances, angles and torsion angles; correlations between e.s.d.'s in cell parameters are only used when they are defined by crystal symmetry. An approximate (isotropic) treatment of cell e.s.d.'s is used for estimating e.s.d.'s involving 1.s. planes.

Refinement. Reflections were merged by SHELXL according to the crystal class for the calculation of statistics and refinement.

Fractional atomic coordinates and isotropic or equivalent isotropic displacement parameters $\left(\AA^{2}\right)$

\begin{tabular}{|c|c|c|c|c|c|}
\hline & $x$ & $y$ & $z$ & $U_{\text {iso }} * / U_{\text {eq }}$ & Occ. $(<1)$ \\
\hline $\mathrm{Br} 1$ & $0.75783(2)$ & $0.69637(2)$ & $0.16055(2)$ & $0.01583(4)$ & \\
\hline $\mathrm{Br} 2$ & $-0.01546(2)$ & $0.16774(2)$ & $0.12333(2)$ & $0.01833(4)$ & \\
\hline $\mathrm{O} 1$ & $1.18246(16)$ & $0.98222(14)$ & $0.33171(13)$ & $0.0166(2)$ & \\
\hline $\mathrm{O} 2$ & $0.62026(15)$ & $0.76781(13)$ & $0.41041(13)$ & $0.0157(2)$ & \\
\hline $\mathrm{H} 2$ & $0.548(5)$ & $0.699(4)$ & $0.323(3)$ & $0.023^{*}$ & $0.49(3)$ \\
\hline $\mathrm{O} 3$ & $-0.34652(16)$ & $0.22816(14)$ & $-0.08651(15)$ & $0.0209(2)$ & \\
\hline $\mathrm{O} 4$ & $0.33771(16)$ & $0.50578(13)$ & $0.19213(13)$ & $0.0164(2)$ & \\
\hline $\mathrm{H} 4$ & $0.427(5)$ & $0.603(3)$ & $0.226(5)$ & $0.025^{*}$ & $0.51(3)$ \\
\hline O5 & $0.82461(16)$ & $0.26388(15)$ & $0.42254(14)$ & $0.0201(2)$ & \\
\hline N1 & 0.48737 (19) & $0.29440(17)$ & $0.32853(17)$ & $0.0170(2)$ & \\
\hline $\mathrm{C} 1$ & $1.0924(2)$ & 0.98617 (17) & $0.40452(17)$ & $0.0123(2)$ & \\
\hline $\mathrm{C} 2$ & $0.8902(2)$ & $0.86739(17)$ & $0.35299(17)$ & $0.0124(2)$ & \\
\hline $\mathrm{C} 3$ & $0.7974(2)$ & $0.87220(17)$ & $0.44093(17)$ & $0.0126(2)$ & \\
\hline $\mathrm{C} 4$ & $-0.1856(2)$ & $0.34950(18)$ & $-0.04567(18)$ & $0.0146(3)$ & \\
\hline $\mathrm{C} 5$ & $-0.0041(2)$ & $0.35821(17)$ & $0.05629(17)$ & $0.0139(3)$ & \\
\hline C6 & $0.1730(2)$ & 0.49677 (18) & $0.10262(17)$ & $0.0145(3)$ & \\
\hline $\mathrm{C} 7$ & $0.6812(2)$ & $0.42756(19)$ & $0.45862(19)$ & $0.0176(3)$ & \\
\hline H7A & 0.6639 & 0.4841 & 0.5393 & $0.021 *$ & \\
\hline $\mathrm{H} 7 \mathrm{~B}$ & 0.7493 & 0.5130 & 0.4132 & $0.021^{*}$ & \\
\hline C8 & $0.8010(2)$ & $0.3481(2)$ & $0.53723(19)$ & $0.0188(3)$ & \\
\hline $\mathrm{H} 8 \mathrm{~A}$ & 0.9304 & 0.4362 & 0.6229 & $0.023 *$ & \\
\hline H8B & 0.7355 & 0.2669 & 0.5872 & $0.023 *$ & \\
\hline $\mathrm{C} 9$ & $0.6388(2)$ & $0.1307(2)$ & $0.3035(2)$ & $0.0206(3)$ & \\
\hline H9A & 0.5750 & 0.0516 & 0.3557 & $0.025^{*}$ & \\
\hline H9B & 0.6567 & 0.0681 & 0.2277 & $0.025^{*}$ & \\
\hline $\mathrm{C} 10$ & $0.5099(2)$ & $0.1976(2)$ & 0.21215 (19) & $0.0196(3)$ & \\
\hline $\mathrm{H} 10 \mathrm{~A}$ & 0.5690 & 0.2717 & 0.1543 & $0.023^{*}$ & \\
\hline H10B & 0.3808 & 0.1032 & 0.1324 & $0.023 *$ & \\
\hline $\mathrm{H} 1 \mathrm{~A}$ & $0.423(3)$ & $0.342(3)$ & $0.277(3)$ & $0.023(5)^{*}$ & \\
\hline H1B & $0.422(3)$ & $0.223(3)$ & $0.367(3)$ & $0.027(6)^{*}$ & \\
\hline
\end{tabular}


Atomic displacement parameters $\left(\AA^{2}\right)$

\begin{tabular}{lllllll}
\hline & $U^{11}$ & $U^{22}$ & $U^{33}$ & $U^{12}$ & $U^{13}$ & $U^{23}$ \\
\hline Br1 & $0.01430(7)$ & $0.01537(7)$ & $0.01367(7)$ & $0.00509(6)$ & $0.00595(5)$ & $0.00068(5)$ \\
Br2 & $0.01566(8)$ & $0.01334(7)$ & $0.02186(8)$ & $0.00595(6)$ & $0.00607(6)$ & $0.00785(6)$ \\
O1 & $0.0150(5)$ & $0.0183(5)$ & $0.0179(5)$ & $0.0075(4)$ & $0.0102(4)$ & $0.0046(4)$ \\
O2 & $0.0100(5)$ & $0.0154(5)$ & $0.0166(5)$ & $0.0031(4)$ & $0.0059(4)$ & $0.0020(4)$ \\
O3 & $0.0129(5)$ & $0.0152(5)$ & $0.0277(6)$ & $0.0040(4)$ & $0.0065(4)$ & $0.0072(4)$ \\
O4 & $0.0122(5)$ & $0.0129(5)$ & $0.0182(5)$ & $0.0048(4)$ & $0.0036(4)$ & $0.0037(4)$ \\
O5 & $0.0151(5)$ & $0.0241(6)$ & $0.0217(5)$ & $0.0117(5)$ & $0.0073(4)$ & $0.0036(4)$ \\
N1 & $0.0139(6)$ & $0.0193(6)$ & $0.0223(6)$ & $0.0101(5)$ & $0.0096(5)$ & $0.0109(5)$ \\
C1 & $0.0117(6)$ & $0.0115(6)$ & $0.0141(6)$ & $0.0063(5)$ & $0.0056(5)$ & $0.0052(5)$ \\
C2 & $0.0109(6)$ & $0.0119(6)$ & $0.0120(6)$ & $0.0047(5)$ & $0.0044(5)$ & $0.0022(5)$ \\
C3 & $0.0106(6)$ & $0.0122(6)$ & $0.0145(6)$ & $0.0064(5)$ & $0.0045(5)$ & $0.0049(5)$ \\
C4 & $0.0152(7)$ & $0.0125(6)$ & $0.0155(6)$ & $0.0060(5)$ & $0.0080(5)$ & $0.0028(5)$ \\
C5 & $0.0147(6)$ & $0.0115(6)$ & $0.0153(6)$ & $0.0062(5)$ & $0.0070(5)$ & $0.0048(5)$ \\
C6 & $0.0158(7)$ & $0.0129(6)$ & $0.0133(6)$ & $0.0058(5)$ & $0.0072(5)$ & $0.0023(5)$ \\
C7 & $0.0180(7)$ & $0.0161(7)$ & $0.0198(7)$ & $0.0090(6)$ & $0.0093(6)$ & $0.0052(6)$ \\
C8 & $0.0170(7)$ & $0.0231(7)$ & $0.0167(7)$ & $0.0118(6)$ & $0.0064(6)$ & $0.0052(6)$ \\
C9 & $0.0189(7)$ & $0.0171(7)$ & $0.0232(8)$ & $0.0099(6)$ & $0.0068(6)$ & $0.0032(6)$ \\
C10 & $0.0174(7)$ & $0.0182(7)$ & $0.0179(7)$ & $0.0086(6)$ & $0.0043(6)$ & $0.0019(6)$ \\
& & & & & & \\
\hline
\end{tabular}

Geometric parameters $\left(\AA,{ }^{\circ}\right)$

\begin{tabular}{|c|c|c|c|}
\hline $\mathrm{Br} 1-\mathrm{C} 2$ & $1.8807(14)$ & $\mathrm{C} 1-\mathrm{C} 3^{\mathrm{i}}$ & 1.5265 (19) \\
\hline $\mathrm{Br} 2-\mathrm{C} 5$ & $1.8767(14)$ & $\mathrm{C} 2-\mathrm{C} 3$ & $1.368(2)$ \\
\hline $\mathrm{O} 1-\mathrm{C} 1$ & $1.2295(18)$ & $\mathrm{C} 4-\mathrm{C} 5$ & $1.445(2)$ \\
\hline $\mathrm{O} 2-\mathrm{C} 3$ & $1.2953(17)$ & $\mathrm{C} 4-\mathrm{C} 6^{\mathrm{ii}}$ & $1.520(2)$ \\
\hline $\mathrm{O} 2-\mathrm{H} 2$ & $0.81(3)$ & $\mathrm{C} 5-\mathrm{C} 6$ & $1.362(2)$ \\
\hline $\mathrm{O} 3-\mathrm{C} 4$ & $1.2229(18)$ & $\mathrm{C} 7-\mathrm{C} 8$ & $1.511(2)$ \\
\hline $\mathrm{O} 4-\mathrm{C} 6$ & $1.3002(18)$ & $\mathrm{C} 7-\mathrm{H} 7 \mathrm{~A}$ & 0.9900 \\
\hline $\mathrm{O} 4-\mathrm{H} 4$ & $0.82(3)$ & $\mathrm{C} 7-\mathrm{H} 7 \mathrm{~B}$ & 0.9900 \\
\hline $\mathrm{O} 5-\mathrm{C} 8$ & $1.4212(19)$ & $\mathrm{C} 8-\mathrm{H} 8 \mathrm{~A}$ & 0.9900 \\
\hline $\mathrm{O} 5-\mathrm{C} 9$ & $1.428(2)$ & $\mathrm{C} 8-\mathrm{H} 8 \mathrm{~B}$ & 0.9900 \\
\hline $\mathrm{N} 1-\mathrm{C} 7$ & $1.492(2)$ & $\mathrm{C} 9-\mathrm{C} 10$ & $1.509(2)$ \\
\hline $\mathrm{N} 1-\mathrm{C} 10$ & $1.494(2)$ & $\mathrm{C} 9-\mathrm{H} 9 \mathrm{~A}$ & 0.9900 \\
\hline $\mathrm{N} 1-\mathrm{H} 1 \mathrm{~A}$ & $0.88(3)$ & C9-H9B & 0.9900 \\
\hline $\mathrm{N} 1-\mathrm{H} 1 \mathrm{~B}$ & $0.86(3)$ & $\mathrm{C} 10-\mathrm{H} 10 \mathrm{~A}$ & 0.9900 \\
\hline $\mathrm{C} 1-\mathrm{C} 2$ & $1.4407(19)$ & $\mathrm{C} 10-\mathrm{H} 10 \mathrm{~B}$ & 0.9900 \\
\hline $\mathrm{C} 3-\mathrm{O} 2-\mathrm{H} 2$ & $118(3)$ & $\mathrm{C} 5-\mathrm{C} 6-\mathrm{C}^{\mathrm{ii}}$ & $120.03(13)$ \\
\hline $\mathrm{C} 6-\mathrm{O} 4-\mathrm{H} 4$ & $111(3)$ & $\mathrm{N} 1-\mathrm{C} 7-\mathrm{C} 8$ & $109.20(12)$ \\
\hline $\mathrm{C} 8-\mathrm{O} 5-\mathrm{C} 9$ & $109.71(12)$ & $\mathrm{N} 1-\mathrm{C} 7-\mathrm{H} 7 \mathrm{~A}$ & 109.8 \\
\hline $\mathrm{C} 7-\mathrm{N} 1-\mathrm{C} 10$ & $110.91(12)$ & $\mathrm{C} 8-\mathrm{C} 7-\mathrm{H} 7 \mathrm{~A}$ & 109.8 \\
\hline $\mathrm{C} 7-\mathrm{N} 1-\mathrm{H} 1 \mathrm{~A}$ & $109.1(14)$ & $\mathrm{N} 1-\mathrm{C} 7-\mathrm{H} 7 \mathrm{~B}$ & 109.8 \\
\hline $\mathrm{C} 10-\mathrm{N} 1-\mathrm{H} 1 \mathrm{~A}$ & $108.4(14)$ & $\mathrm{C} 8-\mathrm{C} 7-\mathrm{H} 7 \mathrm{~B}$ & 109.8 \\
\hline $\mathrm{C} 7-\mathrm{N} 1-\mathrm{H} 1 \mathrm{~B}$ & $111.0(15)$ & $\mathrm{H} 7 \mathrm{~A}-\mathrm{C} 7-\mathrm{H} 7 \mathrm{~B}$ & 108.3 \\
\hline $\mathrm{C} 10-\mathrm{N} 1-\mathrm{H} 1 \mathrm{~B}$ & $107.1(15)$ & $\mathrm{O} 5-\mathrm{C} 8-\mathrm{C} 7$ & $110.61(13)$ \\
\hline
\end{tabular}




\begin{tabular}{|c|c|c|c|}
\hline $\mathrm{H} 1 \mathrm{~A}-\mathrm{N} 1-\mathrm{H} 1 \mathrm{~B}$ & $110(2)$ & $\mathrm{O} 5-\mathrm{C} 8-\mathrm{H} 8 \mathrm{~A}$ & 109.5 \\
\hline $\mathrm{O} 1-\mathrm{C} 1-\mathrm{C} 2$ & $124.43(13)$ & $\mathrm{C} 7-\mathrm{C} 8-\mathrm{H} 8 \mathrm{~A}$ & 109.5 \\
\hline $\mathrm{O} 1-\mathrm{C} 1-\mathrm{C} 3^{\mathrm{i}}$ & $117.51(13)$ & $\mathrm{O} 5-\mathrm{C} 8-\mathrm{H} 8 \mathrm{~B}$ & 109.5 \\
\hline $\mathrm{C} 2-\mathrm{C} 1-\mathrm{C} 3^{\mathrm{i}}$ & $118.06(12)$ & $\mathrm{C} 7-\mathrm{C} 8-\mathrm{H} 8 \mathrm{~B}$ & 109.5 \\
\hline $\mathrm{C} 3-\mathrm{C} 2-\mathrm{C} 1$ & $122.59(13)$ & $\mathrm{H} 8 \mathrm{~A}-\mathrm{C} 8-\mathrm{H} 8 \mathrm{~B}$ & 108.1 \\
\hline $\mathrm{C} 3-\mathrm{C} 2-\mathrm{Br} 1$ & $120.39(11)$ & $\mathrm{O} 5-\mathrm{C} 9-\mathrm{C} 10$ & $111.23(13)$ \\
\hline $\mathrm{C} 1-\mathrm{C} 2-\mathrm{Br} 1$ & $116.98(10)$ & $\mathrm{O} 5-\mathrm{C} 9-\mathrm{H} 9 \mathrm{~A}$ & 109.4 \\
\hline $\mathrm{O} 2-\mathrm{C} 3-\mathrm{C} 2$ & $127.82(13)$ & $\mathrm{C} 10-\mathrm{C} 9-\mathrm{H} 9 \mathrm{~A}$ & 109.4 \\
\hline $\mathrm{O} 2-\mathrm{C} 3-\mathrm{C}^{\mathrm{i}}$ & $112.91(12)$ & $\mathrm{O} 5-\mathrm{C} 9-\mathrm{H} 9 \mathrm{~B}$ & 109.4 \\
\hline $\mathrm{C} 2-\mathrm{C} 3-\mathrm{C}^{\mathrm{i}}$ & $119.28(12)$ & $\mathrm{C} 10-\mathrm{C} 9-\mathrm{H} 9 \mathrm{~B}$ & 109.4 \\
\hline $\mathrm{O} 3-\mathrm{C} 4-\mathrm{C} 5$ & $124.23(14)$ & $\mathrm{H} 9 \mathrm{~A}-\mathrm{C} 9-\mathrm{H} 9 \mathrm{~B}$ & 108.0 \\
\hline $\mathrm{O} 3-\mathrm{C} 4-\mathrm{C}^{\mathrm{ii}}$ & $118.64(13)$ & $\mathrm{N} 1-\mathrm{C} 10-\mathrm{C} 9$ & $108.70(13)$ \\
\hline $\mathrm{C} 5-\mathrm{C} 4-\mathrm{C} 6^{\mathrm{ii}}$ & $117.13(12)$ & $\mathrm{N} 1-\mathrm{C} 10-\mathrm{H} 10 \mathrm{~A}$ & 109.9 \\
\hline $\mathrm{C} 6-\mathrm{C} 5-\mathrm{C} 4$ & $122.84(13)$ & $\mathrm{C} 9-\mathrm{C} 10-\mathrm{H} 10 \mathrm{~A}$ & 109.9 \\
\hline $\mathrm{C} 6-\mathrm{C} 5-\mathrm{Br} 2$ & $119.19(11)$ & $\mathrm{N} 1-\mathrm{C} 10-\mathrm{H} 10 \mathrm{~B}$ & 109.9 \\
\hline $\mathrm{C} 4-\mathrm{C} 5-\mathrm{Br} 2$ & $117.97(10)$ & $\mathrm{C} 9-\mathrm{C} 10-\mathrm{H} 10 \mathrm{~B}$ & 109.9 \\
\hline $\mathrm{O} 4-\mathrm{C} 6-\mathrm{C} 5$ & $123.65(14)$ & $\mathrm{H} 10 \mathrm{~A}-\mathrm{C} 10-\mathrm{H} 10 \mathrm{~B}$ & 108.3 \\
\hline $\mathrm{O} 4-\mathrm{C} 6-\mathrm{C}^{\mathrm{ii}}$ & $116.29(13)$ & & \\
\hline $\mathrm{O} 1-\mathrm{C} 1-\mathrm{C} 2-\mathrm{C} 3$ & $177.29(14)$ & $\mathrm{C} 66^{\mathrm{ii}}-\mathrm{C} 4-\mathrm{C} 5-\mathrm{Br} 2$ & $-178.24(10)$ \\
\hline $\mathrm{C} 3-\mathrm{C} 1-\mathrm{C} 2-\mathrm{C} 3$ & $-3.3(2)$ & $\mathrm{C} 4-\mathrm{C} 5-\mathrm{C} 6-\mathrm{O} 4$ & $-178.83(14)$ \\
\hline $\mathrm{O} 1-\mathrm{C} 1-\mathrm{C} 2-\mathrm{Br} 1$ & $-0.27(19)$ & $\mathrm{Br} 2-\mathrm{C} 5-\mathrm{C} 6-\mathrm{O} 4$ & $-0.1(2)$ \\
\hline $\mathrm{C} 3-\mathrm{C} 1-\mathrm{C} 2-\mathrm{Br} 1$ & $179.12(9)$ & $\mathrm{C} 4-\mathrm{C} 5-\mathrm{C} 6-\mathrm{C} 4^{\mathrm{ii}}$ & $-0.5(2)$ \\
\hline $\mathrm{C} 1-\mathrm{C} 2-\mathrm{C} 3-\mathrm{O} 2$ & $-176.34(14)$ & $\mathrm{Br} 2-\mathrm{C} 5-\mathrm{C} 6-\mathrm{C} 4^{\mathrm{ii}}$ & $178.22(10)$ \\
\hline $\mathrm{Br} 1-\mathrm{C} 2-\mathrm{C} 3-\mathrm{O} 2$ & $1.1(2)$ & $\mathrm{C} 10-\mathrm{N} 1-\mathrm{C} 7-\mathrm{C} 8$ & $-54.86(16)$ \\
\hline $\mathrm{C} 1-\mathrm{C} 2-\mathrm{C} 3-\mathrm{C}^{\mathrm{i}}$ & $3.4(2)$ & $\mathrm{C} 9-\mathrm{O} 5-\mathrm{C} 8-\mathrm{C} 7$ & $-62.32(16)$ \\
\hline $\mathrm{Br} 1-\mathrm{C} 2-\mathrm{C} 3-\mathrm{C} 1^{\mathrm{i}}$ & $-179.16(9)$ & $\mathrm{N} 1-\mathrm{C} 7-\mathrm{C} 8-\mathrm{O} 5$ & $58.54(17)$ \\
\hline $\mathrm{O} 3-\mathrm{C} 4-\mathrm{C} 5-\mathrm{C} 6$ & $-178.49(15)$ & $\mathrm{C} 8-\mathrm{O} 5-\mathrm{C} 9-\mathrm{C} 10$ & $62.50(17)$ \\
\hline $\mathrm{C} 66^{\mathrm{ii}}-\mathrm{C} 4-\mathrm{C} 5-\mathrm{C} 6$ & $0.5(2)$ & $\mathrm{C} 7-\mathrm{N} 1-\mathrm{C} 10-\mathrm{C} 9$ & $54.40(17)$ \\
\hline $\mathrm{O} 3-\mathrm{C} 4-\mathrm{C} 5-\mathrm{Br} 2$ & $2.7(2)$ & $\mathrm{O} 5-\mathrm{C} 9-\mathrm{C} 10-\mathrm{N} 1$ & $-58.02(17)$ \\
\hline
\end{tabular}

Symmetry codes: (i) $-x+2,-y+2,-z+1$; (ii) $-x,-y+1,-z$.

Hydrogen-bond geometry $\left(\AA,{ }^{\circ}\right)$

\begin{tabular}{lllll}
\hline$D-\mathrm{H} \cdots A$ & $D-\mathrm{H}$ & $\mathrm{H} \cdots A$ & $D \cdots A$ & $D-\mathrm{H} \cdots A$ \\
\hline $\mathrm{N} 1-\mathrm{H} 1 A \cdots \mathrm{O} 4$ & $0.88(3)$ & $2.03(3)$ & $2.886(2)$ & $166(2)$ \\
$\mathrm{N} 1-\mathrm{H} 1 B \cdots \mathrm{O} 1^{\text {iii }}$ & $0.86(3)$ & $2.16(3)$ & $2.938(2)$ & $150(2)$ \\
$\mathrm{N} 1-\mathrm{H} 1 B \cdots \mathrm{O} 2^{\text {iv }}$ & $0.86(3)$ & $2.27(3)$ & $2.955(2)$ & $137(2)$ \\
$\mathrm{O} 2-\mathrm{H} 2 \cdots \mathrm{O} 4$ & $0.81(3)$ & $1.77(3)$ & $2.5160(16)$ & $152(4)$ \\
$\mathrm{O} 2-\mathrm{H} 2 \cdots \mathrm{O} 3^{\text {ii }}$ & $0.81(3)$ & $2.57(3)$ & $3.0613(17)$ & $120(3)$ \\
$\mathrm{O} 4-\mathrm{H} 4 \cdots \mathrm{O} 2$ & $0.82(3)$ & $1.82(4)$ & $2.5160(16)$ & $143(4)$ \\
$\mathrm{C} 7-\mathrm{H} 7 A \cdots \mathrm{O} 4{ }^{\text {iv }}$ & 0.99 & 2.53 & $3.391(2)$ & 145 \\
$\mathrm{C} 10-\mathrm{H} 10 B \cdots \mathrm{Br}^{\mathrm{v}}$ & 0.99 & 2.90 & $3.8892(17)$ & 175 \\
\hline
\end{tabular}

Symmetry codes: (ii) $-x,-y+1,-z$; (iii) $x-1, y-1, z$; (iv) $-x+1,-y+1,-z+1$; (v) $-x,-y,-z$. 
(2)

Crystal data

$\mathrm{C}_{4} \mathrm{H}_{10} \mathrm{NO}^{+} \cdot \mathrm{C}_{6} \mathrm{HBr}_{2} \mathrm{O}_{4}^{-}$

$M_{r}=385.01$

Triclinic, $P \overline{1}$

Hall symbol: -P 1

$a=8.62293(18) \AA$

$b=9.21849$ (19) $\AA$

$c=9.4354(2) \AA$

$\alpha=93.5239(7)^{\circ}$

$\beta=112.9190(7)^{\circ}$

$\gamma=115.9777(7)^{\circ}$

$V=596.13(3) \AA^{3}$

Data collection

Rigaku R-AXIS RAPIDII

diffractometer

Detector resolution: 10.000 pixels $\mathrm{mm}^{-1}$

$\omega$ scans

Absorption correction: numerical

(NUMABS; Higashi, 1999)

$T_{\min }=0.098, T_{\max }=0.292$

18176 measured reflections

Refinement

Refinement on $F^{2}$

Least-squares matrix: full

$R\left[F^{2}>2 \sigma\left(F^{2}\right)\right]=0.018$

$w R\left(F^{2}\right)=0.046$

$S=1.10$

3473 reflections

178 parameters

2 restraints
$Z=2$

$F(000)=376.00$

$D_{\mathrm{x}}=2.145 \mathrm{Mg} \mathrm{m}^{-3}$

Mo $K \alpha$ radiation, $\lambda=0.71075 \AA$

Cell parameters from 15916 reflections

$\theta=3.0-30.1^{\circ}$

$\mu=6.83 \mathrm{~mm}^{-1}$

$T=145 \mathrm{~K}$

Block, brown

$0.40 \times 0.34 \times 0.18 \mathrm{~mm}$

3473 independent reflections

3181 reflections with $I>2 \sigma(I)$

$R_{\text {int }}=0.028$

$\theta_{\text {max }}=30.0^{\circ}, \theta_{\text {min }}=3.0^{\circ}$

$h=-12 \rightarrow 12$

$k=-12 \rightarrow 12$

$l=-13 \rightarrow 13$

Hydrogen site location: mixed

$\mathrm{H}$ atoms treated by a mixture of independent and constrained refinement

$w=1 /\left[\sigma^{2}\left(F_{0}^{2}\right)+(0.020 P)^{2}+0.3611 P\right]$

where $P=\left(F_{\mathrm{o}}{ }^{2}+2 F_{\mathrm{c}}{ }^{2}\right) / 3$

$(\Delta / \sigma)_{\max }=0.001$

$\Delta \rho_{\max }=0.48$ e $\AA^{-3}$

$\Delta \rho_{\min }=-0.44$ e $\AA^{-3}$

Special details

Geometry. All e.s.d.'s (except the e.s.d. in the dihedral angle between two 1.s. planes) are estimated using the full covariance matrix. The cell e.s.d.'s are taken into account individually in the estimation of e.s.d.'s in distances, angles and torsion angles; correlations between e.s.d.'s in cell parameters are only used when they are defined by crystal symmetry. An approximate (isotropic) treatment of cell e.s.d.'s is used for estimating e.s.d.'s involving 1.s. planes.

Refinement. Reflections were merged by SHELXL according to the crystal class for the calculation of statistics and refinement.

Fractional atomic coordinates and isotropic or equivalent isotropic displacement parameters $\left(\AA^{2}\right)$

\begin{tabular}{llllll}
\hline & $x$ & $y$ & $z$ & $U_{\text {iso }} * U_{\text {eq }}$ & Occ. $(<1)$ \\
\hline Br1 & $0.75812(2)$ & $0.69658(2)$ & $0.16087(2)$ & $0.01737(4)$ & \\
Br2 & $-0.01570(2)$ & $0.16791(2)$ & $0.12324(2)$ & $0.02003(5)$ & \\
O1 & $1.18243(15)$ & $0.98258(14)$ & $0.33190(13)$ & $0.0179(2)$ & \\
O2 & $0.62059(15)$ & $0.76777(13)$ & $0.41035(13)$ & $0.0172(2)$ & $0.52(3)$ \\
H2 & $0.547(5)$ & $0.698(4)$ & $0.323(3)$ & $0.026^{*}$ & \\
O3 & $-0.34653(16)$ & $0.22841(14)$ & $-0.08661(15)$ & $0.0227(2)$ & \\
O4 & $0.33774(15)$ & $0.50586(13)$ & $0.19208(13)$ & $0.0175(2)$ &
\end{tabular}




\begin{tabular}{|c|c|c|c|c|c|}
\hline $\mathrm{H} 4$ & $0.430(5)$ & $0.603(3)$ & $0.231(5)$ & $0.026^{*}$ & $0.48(3)$ \\
\hline O5 & $0.82422(16)$ & $0.26379(15)$ & $0.42261(14)$ & $0.0219(2)$ & \\
\hline N1 & 0.48734 (19) & $0.29444(17)$ & $0.32852(17)$ & $0.0183(2)$ & \\
\hline $\mathrm{C} 1$ & $1.0922(2)$ & $0.98623(17)$ & $0.40454(17)$ & $0.0131(2)$ & \\
\hline $\mathrm{C} 2$ & $0.8905(2)$ & $0.86761(17)$ & $0.35306(17)$ & $0.0138(2)$ & \\
\hline $\mathrm{C} 3$ & $0.7975(2)$ & $0.87207(17)$ & $0.44087(17)$ & $0.0133(2)$ & \\
\hline $\mathrm{C} 4$ & $-0.1856(2)$ & $0.34979(18)$ & $-0.04558(17)$ & $0.0156(3)$ & \\
\hline $\mathrm{C} 5$ & $-0.0041(2)$ & $0.35843(17)$ & $0.05629(17)$ & $0.0149(3)$ & \\
\hline C6 & $0.1731(2)$ & $0.49685(18)$ & $0.10257(17)$ & $0.0154(3)$ & \\
\hline $\mathrm{C} 7$ & $0.6812(2)$ & 0.42735 (19) & 0.45871 (19) & $0.0191(3)$ & \\
\hline $\mathrm{H} 7 \mathrm{~A}$ & 0.6639 & 0.4839 & 0.5393 & $0.023 *$ & \\
\hline H7B & 0.7495 & 0.5128 & 0.4135 & $0.023 *$ & \\
\hline $\mathrm{C} 8$ & $0.8006(2)$ & $0.3479(2)$ & 0.53709 (19) & $0.0204(3)$ & \\
\hline $\mathrm{H} 8 \mathrm{~A}$ & 0.9300 & 0.4358 & 0.6228 & $0.025^{*}$ & \\
\hline H8B & 0.7349 & 0.2667 & 0.5868 & $0.025^{*}$ & \\
\hline C9 & $0.6387(2)$ & $0.1307(2)$ & $0.3035(2)$ & $0.0227(3)$ & \\
\hline H9A & 0.5748 & 0.0515 & 0.3556 & $0.027 *$ & \\
\hline H9B & 0.6567 & 0.0683 & 0.2278 & $0.027 *$ & \\
\hline $\mathrm{C} 10$ & $0.5097(2)$ & $0.1977(2)$ & $0.21235(19)$ & $0.0212(3)$ & \\
\hline H10A & 0.5687 & 0.2716 & 0.1545 & $0.025^{*}$ & \\
\hline H10B & 0.3806 & 0.1034 & 0.1327 & $0.025 *$ & \\
\hline $\mathrm{H} 1 \mathrm{~A}$ & $0.425(3)$ & $0.342(3)$ & $0.278(3)$ & $0.024(5)^{*}$ & \\
\hline H1B & $0.422(3)$ & $0.224(3)$ & $0.365(3)$ & $0.030(6)^{*}$ & \\
\hline
\end{tabular}

Atomic displacement parameters $\left(\AA^{2}\right)$

\begin{tabular}{lllllll}
\hline & $U^{11}$ & $U^{22}$ & $U^{33}$ & $U^{12}$ & $U^{13}$ & $U^{23}$ \\
\hline Br1 & $0.01574(7)$ & $0.01685(7)$ & $0.01495(7)$ & $0.00556(6)$ & $0.00658(5)$ & $0.00055(5)$ \\
Br2 & $0.01706(8)$ & $0.01455(7)$ & $0.02405(8)$ & $0.00653(6)$ & $0.00668(6)$ & $0.00865(6)$ \\
O1 & $0.0161(5)$ & $0.0199(5)$ & $0.0195(5)$ & $0.0081(4)$ & $0.0110(4)$ & $0.0050(4)$ \\
O2 & $0.0108(5)$ & $0.0164(5)$ & $0.0184(5)$ & $0.0030(4)$ & $0.0064(4)$ & $0.0015(4)$ \\
O3 & $0.0136(5)$ & $0.0158(5)$ & $0.0308(6)$ & $0.0039(4)$ & $0.0068(5)$ & $0.0081(4)$ \\
O4 & $0.0128(5)$ & $0.0138(5)$ & $0.0195(5)$ & $0.0051(4)$ & $0.0035(4)$ & $0.0038(4)$ \\
O5 & $0.0167(5)$ & $0.0258(6)$ & $0.0240(6)$ & $0.0130(5)$ & $0.0079(4)$ & $0.0038(5)$ \\
N1 & $0.0146(6)$ & $0.0210(6)$ & $0.0244(7)$ & $0.0108(5)$ & $0.0105(5)$ & $0.0118(5)$ \\
C1 & $0.0124(6)$ & $0.0127(6)$ & $0.0150(6)$ & $0.0069(5)$ & $0.0061(5)$ & $0.0057(5)$ \\
C2 & $0.0124(6)$ & $0.0134(6)$ & $0.0134(6)$ & $0.0056(5)$ & $0.0051(5)$ & $0.0025(5)$ \\
C3 & $0.0113(6)$ & $0.0125(6)$ & $0.0151(6)$ & $0.0064(5)$ & $0.0046(5)$ & $0.0049(5)$ \\
C4 & $0.0168(7)$ & $0.0131(6)$ & $0.0161(6)$ & $0.0063(5)$ & $0.0083(5)$ & $0.0028(5)$ \\
C5 & $0.0156(6)$ & $0.0123(6)$ & $0.0161(6)$ & $0.0067(5)$ & $0.0071(5)$ & $0.0047(5)$ \\
C6 & $0.0166(7)$ & $0.0139(6)$ & $0.0139(6)$ & $0.0060(5)$ & $0.0076(5)$ & $0.0021(5)$ \\
C7 & $0.0195(7)$ & $0.0172(7)$ & $0.0223(7)$ & $0.0095(6)$ & $0.0107(6)$ & $0.0059(6)$ \\
C8 & $0.0187(7)$ & $0.0251(7)$ & $0.0177(7)$ & $0.0129(6)$ & $0.0066(6)$ & $0.0051(6)$ \\
C9 & $0.0209(7)$ & $0.0185(7)$ & $0.0255(8)$ & $0.0108(6)$ & $0.0073(6)$ & $0.0028(6)$ \\
C10 & $0.0185(7)$ & $0.0206(7)$ & $0.0189(7)$ & $0.0093(6)$ & $0.0047(6)$ & $0.0022(6)$ \\
& & & & & &
\end{tabular}


Geometric parameters $\left(\AA,{ }^{\circ}\right)$

\begin{tabular}{|c|c|c|c|}
\hline $\mathrm{Br} 1-\mathrm{C} 2$ & $1.8807(14)$ & $\mathrm{C} 1-\mathrm{C} 3^{\mathrm{i}}$ & $1.5282(19)$ \\
\hline $\mathrm{Br} 2-\mathrm{C} 5$ & $1.8775(14)$ & $\mathrm{C} 2-\mathrm{C} 3$ & $1.3684(19)$ \\
\hline $\mathrm{O} 1-\mathrm{C} 1$ & $1.2298(17)$ & $\mathrm{C} 4-\mathrm{C} 5$ & $1.446(2)$ \\
\hline $\mathrm{O} 2-\mathrm{C} 3$ & $1.2937(17)$ & $\mathrm{C} 4-\mathrm{C}^{\mathrm{ii}}$ & $1.518(2)$ \\
\hline $\mathrm{O} 2-\mathrm{H} 2$ & $0.82(3)$ & $\mathrm{C} 5-\mathrm{C} 6$ & $1.363(2)$ \\
\hline $\mathrm{O} 3-\mathrm{C} 4$ & $1.2231(18)$ & $\mathrm{C} 7-\mathrm{C} 8$ & $1.509(2)$ \\
\hline $\mathrm{O} 4-\mathrm{C} 6$ & $1.2997(18)$ & $\mathrm{C} 7-\mathrm{H} 7 \mathrm{~A}$ & 0.9900 \\
\hline $\mathrm{O} 4-\mathrm{H} 4$ & $0.82(3)$ & $\mathrm{C} 7-\mathrm{H} 7 \mathrm{~B}$ & 0.9900 \\
\hline $\mathrm{O} 5-\mathrm{C} 8$ & $1.4201(19)$ & $\mathrm{C} 8-\mathrm{H} 8 \mathrm{~A}$ & 0.9900 \\
\hline $\mathrm{O} 5-\mathrm{C} 9$ & $1.428(2)$ & $\mathrm{C} 8-\mathrm{H} 8 \mathrm{~B}$ & 0.9900 \\
\hline $\mathrm{N} 1-\mathrm{C} 7$ & $1.492(2)$ & $\mathrm{C} 9-\mathrm{C} 10$ & $1.510(2)$ \\
\hline $\mathrm{N} 1-\mathrm{C} 10$ & $1.493(2)$ & C9-H9A & 0.9900 \\
\hline $\mathrm{N} 1-\mathrm{H} 1 \mathrm{~A}$ & $0.86(3)$ & C9-H9B & 0.9900 \\
\hline $\mathrm{N} 1-\mathrm{H} 1 \mathrm{~B}$ & $0.85(3)$ & $\mathrm{C} 10-\mathrm{H} 10 \mathrm{~A}$ & 0.9900 \\
\hline $\mathrm{C} 1-\mathrm{C} 2$ & $1.4377(19)$ & $\mathrm{C} 10-\mathrm{H} 10 \mathrm{~B}$ & 0.9900 \\
\hline $\mathrm{C} 3-\mathrm{O} 2-\mathrm{H} 2$ & $118(3)$ & $\mathrm{C} 5-\mathrm{C} 6-\mathrm{C}^{\mathrm{ii}}$ & $119.95(13)$ \\
\hline $\mathrm{C} 6-\mathrm{O} 4-\mathrm{H} 4$ & $113(3)$ & $\mathrm{N} 1-\mathrm{C} 7-\mathrm{C} 8$ & $109.23(12)$ \\
\hline $\mathrm{C} 8-\mathrm{O} 5-\mathrm{C} 9$ & $109.77(12)$ & $\mathrm{N} 1-\mathrm{C} 7-\mathrm{H} 7 \mathrm{~A}$ & 109.8 \\
\hline $\mathrm{C} 7-\mathrm{N} 1-\mathrm{C} 10$ & $110.93(12)$ & $\mathrm{C} 8-\mathrm{C} 7-\mathrm{H} 7 \mathrm{~A}$ & 109.8 \\
\hline $\mathrm{C} 7-\mathrm{N} 1-\mathrm{H} 1 \mathrm{~A}$ & $108.5(14)$ & $\mathrm{N} 1-\mathrm{C} 7-\mathrm{H} 7 \mathrm{~B}$ & 109.8 \\
\hline $\mathrm{C} 10-\mathrm{N} 1-\mathrm{H} 1 \mathrm{~A}$ & $108.6(14)$ & $\mathrm{C} 8-\mathrm{C} 7-\mathrm{H} 7 \mathrm{~B}$ & 109.8 \\
\hline $\mathrm{C} 7-\mathrm{N} 1-\mathrm{H} 1 \mathrm{~B}$ & $111.9(16)$ & $\mathrm{H} 7 \mathrm{~A}-\mathrm{C} 7-\mathrm{H} 7 \mathrm{~B}$ & 108.3 \\
\hline $\mathrm{C} 10-\mathrm{N} 1-\mathrm{H} 1 \mathrm{~B}$ & $106.6(15)$ & $\mathrm{O} 5-\mathrm{C} 8-\mathrm{C} 7$ & $110.62(13)$ \\
\hline $\mathrm{H} 1 \mathrm{~A}-\mathrm{N} 1-\mathrm{H} 1 \mathrm{~B}$ & $110(2)$ & $\mathrm{O} 5-\mathrm{C} 8-\mathrm{H} 8 \mathrm{~A}$ & 109.5 \\
\hline $\mathrm{O} 1-\mathrm{C} 1-\mathrm{C} 2$ & $124.48(13)$ & $\mathrm{C} 7-\mathrm{C} 8-\mathrm{H} 8 \mathrm{~A}$ & 109.5 \\
\hline $\mathrm{O} 1-\mathrm{C} 1-\mathrm{C} 3^{\mathrm{i}}$ & $117.39(12)$ & $\mathrm{O} 5-\mathrm{C} 8-\mathrm{H} 8 \mathrm{~B}$ & 109.5 \\
\hline $\mathrm{C} 2-\mathrm{C} 1-\mathrm{C} 3^{\mathrm{i}}$ & $118.13(12)$ & $\mathrm{C} 7-\mathrm{C} 8-\mathrm{H} 8 \mathrm{~B}$ & 109.5 \\
\hline $\mathrm{C} 3-\mathrm{C} 2-\mathrm{C} 1$ & $122.62(13)$ & $\mathrm{H} 8 \mathrm{~A}-\mathrm{C} 8-\mathrm{H} 8 \mathrm{~B}$ & 108.1 \\
\hline $\mathrm{C} 3-\mathrm{C} 2-\mathrm{Br} 1$ & $120.30(11)$ & $\mathrm{O} 5-\mathrm{C} 9-\mathrm{C} 10$ & $111.19(13)$ \\
\hline $\mathrm{C} 1-\mathrm{C} 2-\mathrm{Br} 1$ & $117.03(10)$ & $\mathrm{O} 5-\mathrm{C} 9-\mathrm{H} 9 \mathrm{~A}$ & 109.4 \\
\hline $\mathrm{O} 2-\mathrm{C} 3-\mathrm{C} 2$ & $127.87(13)$ & $\mathrm{C} 10-\mathrm{C} 9-\mathrm{H} 9 \mathrm{~A}$ & 109.4 \\
\hline $\mathrm{O} 2-\mathrm{C} 3-\mathrm{C} 1^{\mathrm{i}}$ & $112.96(12)$ & $\mathrm{O} 5-\mathrm{C} 9-\mathrm{H} 9 \mathrm{~B}$ & 109.4 \\
\hline $\mathrm{C} 2-\mathrm{C} 3-\mathrm{C} 1^{\mathrm{i}}$ & $119.17(12)$ & $\mathrm{C} 10-\mathrm{C} 9-\mathrm{H} 9 \mathrm{~B}$ & 109.4 \\
\hline $\mathrm{O} 3-\mathrm{C} 4-\mathrm{C} 5$ & $124.23(13)$ & $\mathrm{H} 9 \mathrm{~A}-\mathrm{C} 9-\mathrm{H} 9 \mathrm{~B}$ & 108.0 \\
\hline $\mathrm{O} 3-\mathrm{C} 4-\mathrm{C}^{\mathrm{ii}}$ & $118.58(13)$ & $\mathrm{N} 1-\mathrm{C} 10-\mathrm{C} 9$ & $108.74(13)$ \\
\hline $\mathrm{C} 5-\mathrm{C} 4-\mathrm{C} 6^{\mathrm{ii}}$ & $117.19(12)$ & $\mathrm{N} 1-\mathrm{C} 10-\mathrm{H} 10 \mathrm{~A}$ & 109.9 \\
\hline $\mathrm{C} 6-\mathrm{C} 5-\mathrm{C} 4$ & $122.86(13)$ & $\mathrm{C} 9-\mathrm{C} 10-\mathrm{H} 10 \mathrm{~A}$ & 109.9 \\
\hline $\mathrm{C} 6-\mathrm{C} 5-\mathrm{Br} 2$ & $119.20(11)$ & $\mathrm{N} 1-\mathrm{C} 10-\mathrm{H} 10 \mathrm{~B}$ & 109.9 \\
\hline $\mathrm{C} 4-\mathrm{C} 5-\mathrm{Br} 2$ & $117.92(10)$ & $\mathrm{C} 9-\mathrm{C} 10-\mathrm{H} 10 \mathrm{~B}$ & 109.9 \\
\hline $\mathrm{O} 4-\mathrm{C} 6-\mathrm{C} 5$ & $123.68(13)$ & $\mathrm{H} 10 \mathrm{~A}-\mathrm{C} 10-\mathrm{H} 10 \mathrm{~B}$ & 108.3 \\
\hline $\mathrm{O} 4-\mathrm{C} 6-\mathrm{C}^{\mathrm{ii}}$ & $116.36(12)$ & & \\
\hline $\mathrm{O} 1-\mathrm{C} 1-\mathrm{C} 2-\mathrm{C} 3$ & $177.25(14)$ & $\mathrm{C} 6{ }^{\mathrm{ii}}-\mathrm{C} 4-\mathrm{C} 5-\mathrm{Br} 2$ & $-178.23(10)$ \\
\hline $\mathrm{C} 3^{\mathrm{i}}-\mathrm{C} 1-\mathrm{C} 2-\mathrm{C} 3$ & $-3.4(2)$ & $\mathrm{C} 4-\mathrm{C} 5-\mathrm{C} 6-\mathrm{O} 4$ & $-178.92(13)$ \\
\hline $\mathrm{O} 1-\mathrm{C} 1-\mathrm{C} 2-\mathrm{Br} 1$ & $-0.21(19)$ & $\mathrm{Br} 2-\mathrm{C} 5-\mathrm{C} 6-\mathrm{O} 4$ & $-0.2(2)$ \\
\hline
\end{tabular}




\begin{tabular}{|c|c|c|c|}
\hline $\mathrm{C} 3-\mathrm{C} 1-\mathrm{C} 2-\mathrm{Br} 1$ & $179.18(9)$ & $\mathrm{C} 4-\mathrm{C} 5-\mathrm{C} 6-\mathrm{C} 44^{\mathrm{ii}}$ & $-0.6(2)$ \\
\hline $\mathrm{C} 1-\mathrm{C} 2-\mathrm{C} 3-\mathrm{O} 2$ & $-176.36(13)$ & $\mathrm{Br} 2-\mathrm{C} 5-\mathrm{C} 6-\mathrm{C}^{\mathrm{ii}}$ & $178.19(10)$ \\
\hline $\mathrm{Br} 1-\mathrm{C} 2-\mathrm{C} 3-\mathrm{O} 2$ & $1.0(2)$ & $\mathrm{C} 10-\mathrm{N} 1-\mathrm{C} 7-\mathrm{C} 8$ & $-54.81(16)$ \\
\hline $\mathrm{C} 1-\mathrm{C} 2-\mathrm{C} 3-\mathrm{C}^{\mathrm{i}}$ & $3.4(2)$ & $\mathrm{C} 9-\mathrm{O} 5-\mathrm{C} 8-\mathrm{C} 7$ & $-62.38(16)$ \\
\hline $\mathrm{Br} 1-\mathrm{C} 2-\mathrm{C} 3-\mathrm{C}^{\mathrm{i}}$ & $-179.22(9)$ & $\mathrm{N} 1-\mathrm{C} 7-\mathrm{C} 8-\mathrm{O} 5$ & $58.53(17)$ \\
\hline $\mathrm{O} 3-\mathrm{C} 4-\mathrm{C} 5-\mathrm{C} 6$ & $-178.65(15)$ & $\mathrm{C} 8-\mathrm{O} 5-\mathrm{C} 9-\mathrm{C} 10$ & $62.43(17)$ \\
\hline $\mathrm{C} 6{ }^{\mathrm{ii}}-\mathrm{C} 4-\mathrm{C} 5-\mathrm{C} 6$ & $0.5(2)$ & $\mathrm{C} 7-\mathrm{N} 1-\mathrm{C} 10-\mathrm{C} 9$ & $54.27(17)$ \\
\hline $\mathrm{O} 3-\mathrm{C} 4-\mathrm{C} 5-\mathrm{Br} 2$ & $2.6(2)$ & $\mathrm{O} 5-\mathrm{C} 9-\mathrm{C} 10-\mathrm{N} 1$ & $-57.89(18)$ \\
\hline
\end{tabular}

Symmetry codes: (i) $-x+2,-y+2,-z+1$; (ii) $-x,-y+1,-z$.

Hydrogen-bond geometry $\left(\AA,{ }^{\circ}\right)$

\begin{tabular}{lllll}
\hline$D-\mathrm{H} \cdots A$ & $D-\mathrm{H}$ & $\mathrm{H} \cdots A$ & $D \cdots A$ & $D-\mathrm{H} \cdots A$ \\
\hline $\mathrm{N} 1-\mathrm{H} 1 A \cdots \mathrm{O} 4$ & $0.86(3)$ & $2.04(3)$ & $2.888(2)$ & $166(2)$ \\
$\mathrm{N} 1-\mathrm{H} 1 B \cdots \mathrm{O} 1^{\text {iii }}$ & $0.85(3)$ & $2.17(3)$ & $2.938(2)$ & $151(2)$ \\
$\mathrm{N} 1-\mathrm{H} 1 B \cdots \mathrm{O} 2^{\text {iv }}$ & $0.85(3)$ & $2.29(3)$ & $2.959(2)$ & $136(2)$ \\
$\mathrm{O} 2-\mathrm{H} 2 \cdots \mathrm{O} 4$ & $0.82(3)$ & $1.77(3)$ & $2.5174(16)$ & $153(4)$ \\
$\mathrm{O} 2-\mathrm{H} 2 \cdots \mathrm{O} 3^{\mathrm{ii}}$ & $0.82(3)$ & $2.58(3)$ & $3.0628(17)$ & $120(3)$ \\
$\mathrm{O} 4-\mathrm{H} 4 \cdots \mathrm{O} 2$ & $0.82(3)$ & $1.79(4)$ & $2.5174(16)$ & $147(4)$ \\
$\mathrm{C} 7-\mathrm{H} 7 A \cdots \mathrm{O} 4^{\text {iv }}$ & 0.99 & 2.54 & $3.394(2)$ & 145 \\
$\mathrm{C} 10-\mathrm{H} 10 B \cdots \mathrm{Br} 2^{\mathrm{v}}$ & 0.99 & 2.90 & $3.8905(17)$ & 175
\end{tabular}

Symmetry codes: (ii) $-x,-y+1,-z$; (iii) $x-1, y-1, z$; (iv) $-x+1,-y+1,-z+1$; (v) $-x,-y,-z$.

(3)

Crystal data

$\mathrm{C}_{4} \mathrm{H}_{10} \mathrm{NO}^{+} \cdot \mathrm{C}_{6} \mathrm{HBr}_{2} \mathrm{O}_{4}^{-}$

$M_{r}=385.01$

Triclinic, $P \overline{1}$

Hall symbol: -P 1

$a=8.62824$ (17) $\AA$

$b=9.23087(18) \AA$

$c=9.46007(19) \AA$

$\alpha=93.5321$ (7) ${ }^{\circ}$

$\beta=112.9738(7)^{\circ}$

$\gamma=115.9508(7)^{\circ}$

$V=598.67(3) \AA^{3}$

\section{Data collection}

Rigaku R-AXIS RAPIDII diffractometer

Detector resolution: 10.000 pixels $\mathrm{mm}^{-1}$

$\omega$ scans

Absorption correction: numerical

(NUMABS; Higashi, 1999)

$T_{\min }=0.098, T_{\max }=0.294$

18199 measured reflections

\section{Refinement}

Refinement on $F^{2}$

Least-squares matrix: full
$Z=2$

$F(000)=376.00$

$D_{\mathrm{x}}=2.136 \mathrm{Mg} \mathrm{m}^{-3}$

Mo $K \alpha$ radiation, $\lambda=0.71075 \AA$

Cell parameters from 15838 reflections

$\theta=3.0-30.1^{\circ}$

$\mu=6.80 \mathrm{~mm}^{-1}$

$T=180 \mathrm{~K}$

Block, brown

$0.40 \times 0.34 \times 0.18 \mathrm{~mm}$

3487 independent reflections

3188 reflections with $I>2 \sigma(I)$

$R_{\text {int }}=0.026$

$\theta_{\text {max }}=30.0^{\circ}$

$h=-12 \rightarrow 12$

$k=-12 \rightarrow 12$

$l=-13 \rightarrow 13$

$R\left[F^{2}>2 \sigma\left(F^{2}\right)\right]=0.019$

$w R\left(F^{2}\right)=0.048$ 
$S=1.09$

3487 reflections

178 parameters

2 restraints

Hydrogen site location: mixed
$\mathrm{H}$ atoms treated by a mixture of independent and constrained refinement

$w=1 /\left[\sigma^{2}\left(F_{\mathrm{o}}^{2}\right)+(0.0216 P)^{2}+0.3489 P\right]$ where $P=\left(F_{\mathrm{o}}{ }^{2}+2 F_{\mathrm{c}}{ }^{2}\right) / 3$

$(\Delta / \sigma)_{\max }=0.001$

$\Delta \rho_{\max }=0.59 \mathrm{e} \AA^{-3}$

$\Delta \rho_{\min }=-0.45$ e $\AA^{-3}$

Special details

Geometry. All e.s.d.'s (except the e.s.d. in the dihedral angle between two 1.s. planes) are estimated using the full covariance matrix. The cell e.s.d.'s are taken into account individually in the estimation of e.s.d.'s in distances, angles and torsion angles; correlations between e.s.d.'s in cell parameters are only used when they are defined by crystal symmetry. An approximate (isotropic) treatment of cell e.s.d.'s is used for estimating e.s.d.'s involving 1.s. planes.

Refinement. Reflections were merged by SHELXL according to the crystal class for the calculation of statistics and refinement.

Fractional atomic coordinates and isotropic or equivalent isotropic displacement parameters $\left(\hat{A}^{2}\right)$

\begin{tabular}{|c|c|c|c|c|c|}
\hline & $x$ & $y$ & $z$ & $U_{\text {iso }} * / U_{\text {eq }}$ & Occ. $(<1)$ \\
\hline Br1 & $0.75874(2)$ & $0.69709(2)$ & $0.16160(2)$ & $0.02126(5)$ & \\
\hline $\mathrm{Br} 2$ & $-0.01629(2)$ & $0.16834(2)$ & $0.12298(2)$ & $0.02440(5)$ & \\
\hline $\mathrm{O} 1$ & $1.18225(16)$ & $0.98340(14)$ & $0.33207(13)$ & $0.0212(2)$ & \\
\hline $\mathrm{O} 2$ & $0.62107(15)$ & $0.76749(14)$ & $0.41043(13)$ & $0.0205(2)$ & \\
\hline $\mathrm{H} 2$ & $0.550(6)$ & $0.702(5)$ & $0.321(3)$ & $0.031^{*}$ & $0.50(3)$ \\
\hline $\mathrm{O} 3$ & $-0.34638(16)$ & $0.22923(14)$ & $-0.08655(16)$ & $0.0275(2)$ & \\
\hline $\mathrm{O} 4$ & $0.33726(16)$ & $0.50565(14)$ & $0.19202(13)$ & $0.0209(2)$ & \\
\hline H4 & $0.426(5)$ & $0.604(3)$ & $0.231(5)$ & $0.031 *$ & $0.50(3)$ \\
\hline O5 & $0.82325(17)$ & $0.26349(16)$ & $0.42251(15)$ & $0.0266(2)$ & \\
\hline N1 & 0.48719 (19) & $0.29464(18)$ & $0.32897(17)$ & $0.0216(3)$ & \\
\hline $\mathrm{C} 1$ & $1.0921(2)$ & $0.98669(17)$ & $0.40464(17)$ & $0.0155(2)$ & \\
\hline $\mathrm{C} 2$ & $0.8904(2)$ & $0.86771(17)$ & $0.35338(17)$ & $0.0158(2)$ & \\
\hline $\mathrm{C} 3$ & $0.7977(2)$ & $0.87190(17)$ & $0.44099(17)$ & $0.0157(2)$ & \\
\hline $\mathrm{C} 4$ & $-0.1853(2)$ & $0.35010(18)$ & $-0.04556(18)$ & $0.0185(3)$ & \\
\hline $\mathrm{C} 5$ & $-0.0045(2)$ & $0.35849(17)$ & $0.05607(17)$ & $0.0175(3)$ & \\
\hline C6 & $0.1728(2)$ & $0.49667(18)$ & $0.10245(17)$ & $0.0180(3)$ & \\
\hline $\mathrm{C} 7$ & $0.6809(2)$ & $0.42714(19)$ & $0.4585(2)$ & $0.0227(3)$ & \\
\hline $\mathrm{H} 7 \mathrm{~A}$ & 0.6639 & 0.4840 & 0.5389 & $0.027^{*}$ & \\
\hline H7B & 0.7492 & 0.5122 & 0.4133 & $0.027 *$ & \\
\hline $\mathrm{C} 8$ & $0.7997(2)$ & $0.3474(2)$ & $0.53683(19)$ & $0.0250(3)$ & \\
\hline $\mathrm{H} 8 \mathrm{~A}$ & 0.9291 & 0.4350 & 0.6226 & $0.030^{*}$ & \\
\hline H8B & 0.7337 & 0.2662 & 0.5861 & $0.030^{*}$ & \\
\hline C9 & $0.6381(2)$ & $0.1310(2)$ & $0.3036(2)$ & $0.0272(3)$ & \\
\hline H9A & 0.5742 & 0.0517 & 0.3552 & $0.033^{*}$ & \\
\hline H9B & 0.6562 & 0.0690 & 0.2279 & $0.033^{*}$ & \\
\hline $\mathrm{C} 10$ & $0.5094(2)$ & $0.1980(2)$ & $0.21303(19)$ & $0.0252(3)$ & \\
\hline $\mathrm{H} 10 \mathrm{~A}$ & 0.5683 & 0.2719 & 0.1554 & $0.030^{*}$ & \\
\hline H10B & 0.3803 & 0.1040 & 0.1335 & $0.030^{*}$ & \\
\hline $\mathrm{H} 1 \mathrm{~A}$ & $0.424(3)$ & $0.344(3)$ & $0.277(2)$ & $0.025(5)^{*}$ & \\
\hline H1B & $0.422(3)$ & 0.223 & $0.367(3)$ & $0.026(5)^{*}$ & \\
\hline
\end{tabular}


Atomic displacement parameters $\left(\AA^{2}\right)$

\begin{tabular}{lllllll}
\hline & $U^{11}$ & $U^{22}$ & $U^{33}$ & $U^{12}$ & $U^{13}$ & $U^{23}$ \\
\hline Br1 & $0.01905(8)$ & $0.02063(8)$ & $0.01805(7)$ & $0.00654(6)$ & $0.00783(6)$ & $0.00043(5)$ \\
Br2 & $0.02061(8)$ & $0.01771(7)$ & $0.02936(9)$ & $0.00800(6)$ & $0.00791(6)$ & $0.01079(6)$ \\
O1 & $0.0192(5)$ & $0.0234(5)$ & $0.0227(5)$ & $0.0093(4)$ & $0.0131(4)$ & $0.0057(4)$ \\
O2 & $0.0128(5)$ & $0.0201(5)$ & $0.0215(5)$ & $0.0035(4)$ & $0.0076(4)$ & $0.0019(4)$ \\
O3 & $0.0161(5)$ & $0.0194(5)$ & $0.0374(7)$ & $0.0049(4)$ & $0.0080(5)$ & $0.0101(5)$ \\
O4 & $0.0151(5)$ & $0.0166(5)$ & $0.0230(5)$ & $0.0061(4)$ & $0.0039(4)$ & $0.0046(4)$ \\
O5 & $0.0192(5)$ & $0.0314(6)$ & $0.0295(6)$ & $0.0151(5)$ & $0.0094(5)$ & $0.0045(5)$ \\
N1 & $0.0171(6)$ & $0.0245(6)$ & $0.0290(7)$ & $0.0129(5)$ & $0.0123(5)$ & $0.0141(6)$ \\
C1 & $0.0148(6)$ & $0.0147(6)$ & $0.0180(6)$ & $0.0076(5)$ & $0.0079(5)$ & $0.0069(5)$ \\
C2 & $0.0141(6)$ & $0.0144(6)$ & $0.0159(6)$ & $0.0057(5)$ & $0.0062(5)$ & $0.0027(5)$ \\
C3 & $0.0133(6)$ & $0.0155(6)$ & $0.0180(6)$ & $0.0076(5)$ & $0.0064(5)$ & $0.0061(5)$ \\
C4 & $0.0181(7)$ & $0.0155(6)$ & $0.0197(6)$ & $0.0065(5)$ & $0.0092(5)$ & $0.0034(5)$ \\
C5 & $0.0175(6)$ & $0.0141(6)$ & $0.0192(6)$ & $0.0073(5)$ & $0.0078(5)$ & $0.0053(5)$ \\
C6 & $0.0188(7)$ & $0.0164(6)$ & $0.0166(6)$ & $0.0071(5)$ & $0.0086(5)$ & $0.0030(5)$ \\
C7 & $0.0231(7)$ & $0.0208(7)$ & $0.0259(7)$ & $0.0111(6)$ & $0.0129(6)$ & $0.0066(6)$ \\
C8 & $0.0219(7)$ & $0.0308(8)$ & $0.0218(7)$ & $0.0150(7)$ & $0.0080(6)$ & $0.0061(6)$ \\
C9 & $0.0250(8)$ & $0.0217(7)$ & $0.0317(8)$ & $0.0129(7)$ & $0.0095(7)$ & $0.0036(6)$ \\
C10 & $0.0218(7)$ & $0.0243(7)$ & $0.0220(7)$ & $0.0108(6)$ & $0.0051(6)$ & $0.0025(6)$ \\
& & & & & &
\end{tabular}

Geometric parameters $\left(\AA,{ }^{\circ}\right)$

\begin{tabular}{llll}
\hline $\mathrm{Br} 1-\mathrm{C} 2$ & $1.8803(14)$ & $\mathrm{C} 1-\mathrm{C} 3^{\mathrm{i}}$ & $1.5283(19)$ \\
$\mathrm{Br} 2-\mathrm{C} 5$ & $1.8769(14)$ & $\mathrm{C} 2-\mathrm{C} 3$ & $1.367(2)$ \\
$\mathrm{O} 1-\mathrm{C} 1$ & $1.2299(17)$ & $\mathrm{C} 4-\mathrm{C} 5$ & $1.442(2)$ \\
$\mathrm{O} 2-\mathrm{C} 3$ & $1.2931(17)$ & $\mathrm{C} 4-\mathrm{C} 6^{6 i}$ & $1.519(2)$ \\
$\mathrm{O} 2-\mathrm{H} 2$ & $0.82(3)$ & $\mathrm{C} 5-\mathrm{C} 6$ & $1.363(2)$ \\
$\mathrm{O} 3-\mathrm{C} 4$ & $1.222(18)$ & $\mathrm{C} 7-\mathrm{C} 8$ & $1.509(2)$ \\
$\mathrm{O} 4-\mathrm{C} 6$ & $1.2997(18)$ & $\mathrm{C} 7-\mathrm{H} 7 \mathrm{~A}$ & 0.9900 \\
$\mathrm{O} 4-\mathrm{H} 4$ & $0.82(3)$ & $\mathrm{C} 7-\mathrm{H} 7 \mathrm{~B}$ & 0.9900 \\
$\mathrm{O} 5-\mathrm{C} 8$ & $1.420(2)$ & $\mathrm{C} 8-\mathrm{H} 8 \mathrm{~A}$ & 0.9900 \\
$\mathrm{O} 5-\mathrm{C} 9$ & $1.425(2)$ & $\mathrm{C} 8-\mathrm{H} 8 \mathrm{~B}$ & 0.9900 \\
$\mathrm{~N} 1-\mathrm{C} 7$ & $1.490(2)$ & $\mathrm{C} 9-\mathrm{C} 10$ & $1.508(2)$ \\
$\mathrm{N} 1-\mathrm{C} 10$ & $1.493(2)$ & $\mathrm{C} 9-\mathrm{H} 9 \mathrm{~A}$ & 0.9900 \\
$\mathrm{~N} 1-\mathrm{H} 1 \mathrm{~A}$ & $0.89(3)$ & $\mathrm{C} 9-\mathrm{H} 9 \mathrm{~B}$ & 0.9900 \\
$\mathrm{~N} 1-\mathrm{H} 1 \mathrm{~B}$ & $0.86(3)$ & $\mathrm{C} 10-\mathrm{H} 10 \mathrm{~A}$ & 0.9900 \\
$\mathrm{C} 1-\mathrm{C} 2$ & $1.4394(19)$ & $\mathrm{C} 10-\mathrm{H} 10 \mathrm{~B}$ & 0.9900 \\
& & & $119.94(13)$ \\
$\mathrm{C} 3-\mathrm{O} 2-\mathrm{H} 2$ & $117(3)$ & $\mathrm{C} 5-\mathrm{C} 6-\mathrm{C} 4^{\mathrm{ii}}$ & $109.14(13)$ \\
$\mathrm{C} 6-\mathrm{O} 4-\mathrm{H} 4$ & $111(3)$ & $\mathrm{N} 1-\mathrm{C} 7-\mathrm{C} 8$ & 109.9 \\
$\mathrm{C} 8-\mathrm{O} 5-\mathrm{C} 9$ & $109.83(12)$ & $\mathrm{N} 1-\mathrm{C} 7-\mathrm{H} 7 \mathrm{C} 7-\mathrm{C} 7 \mathrm{~A}$ & 109.9 \\
$\mathrm{C} 7-\mathrm{N} 1-\mathrm{C} 10$ & $110.85(12)$ & $\mathrm{N} 1-\mathrm{C} 7-\mathrm{H} 7 \mathrm{~B}$ & 109.9 \\
$\mathrm{C} 7-\mathrm{N} 1-\mathrm{H} 1 \mathrm{~A}$ & $108.4(14)$ & $\mathrm{C} 8-\mathrm{C} 7-\mathrm{H} 7 \mathrm{~B}$ & 109.9 \\
$\mathrm{C} 10-\mathrm{N} 1-\mathrm{H} 1 \mathrm{~A}$ & $108.2(14)$ & $\mathrm{H} 7 \mathrm{C}-\mathrm{C} 7-\mathrm{H} 7-\mathrm{C} 7$ & 108.3 \\
$\mathrm{C} 7-\mathrm{N} 1-\mathrm{H} 1 \mathrm{~B}$ & $111.2(14)$ & & $110.51(13)$ \\
$\mathrm{C} 10-\mathrm{N} 1-\mathrm{H} 1 \mathrm{~B}$ & $106.4(14)$ & &
\end{tabular}




$\begin{array}{ll}\mathrm{H} 1 \mathrm{~A}-\mathrm{N} 1-\mathrm{H} 1 \mathrm{~B} & 112(2) \\ \mathrm{O} 1-\mathrm{C} 1-\mathrm{C} 2 & 124.51(13) \\ \mathrm{O} 1-\mathrm{C} 1-\mathrm{C} 3^{\mathrm{i}} & 117.41(12) \\ \mathrm{C} 2-\mathrm{C} 1-\mathrm{C} 3^{\mathrm{i}} & 118.08(12) \\ \mathrm{C} 3-\mathrm{C} 2-\mathrm{C} 1 & 122.63(13) \\ \mathrm{C} 3-\mathrm{C} 2-\mathrm{Br} 1 & 120.43(11) \\ \mathrm{C} 1-\mathrm{C} 2-\mathrm{Br} 1 & 116.90(10) \\ \mathrm{O} 2-\mathrm{C} 3-\mathrm{C} 2 & 127.81(13) \\ \mathrm{O} 2-\mathrm{C} 3-\mathrm{C} 1^{\mathrm{i}} & 112.98(12) \\ \mathrm{C} 2-\mathrm{C} 3-\mathrm{C} 1^{\mathrm{i}} & 119.21(12) \\ \mathrm{O} 3-\mathrm{C} 4-\mathrm{C} 5 & 124.27(14) \\ \mathrm{O} 3-\mathrm{C} 4-\mathrm{C} 6{ }^{\mathrm{ii}} & 118.48(14) \\ \mathrm{C} 5-\mathrm{C} 4-\mathrm{C} 6^{\mathrm{ii}} & 117.24(12) \\ \mathrm{C} 6-\mathrm{C} 5-\mathrm{C} 4 & 122.82(13) \\ \mathrm{C} 6-\mathrm{C} 5-\mathrm{Br} 2 & 119.20(11) \\ \mathrm{C} 4-\mathrm{C} 5-\mathrm{Br} 2 & 117.98(10) \\ \mathrm{O} 4-\mathrm{C} 6-\mathrm{C} 5 & 123.69(14) \\ \mathrm{O} 4-\mathrm{C} 6-\mathrm{C} 4^{\mathrm{ii}} & 116.35(13) \\ & \\ \mathrm{O} 1-\mathrm{C} 1-\mathrm{C} 2-\mathrm{C} 3 & 177.30(14) \\ \mathrm{C} 3-\mathrm{C} 1-\mathrm{C} 2-\mathrm{C} 3 & -3.3(2) \\ \mathrm{O} 1-\mathrm{C} 1-\mathrm{C} 2-\mathrm{Br} 1 & -0.20(19) \\ \mathrm{C} 3-\mathrm{C} 1-\mathrm{C} 2-\mathrm{Br} 1 & 179.24(9) \\ \mathrm{C} 1-\mathrm{C} 2-\mathrm{C} 3-\mathrm{O} 2 & -176.48(14) \\ \mathrm{Br} 1-\mathrm{C} 2-\mathrm{C} 3-\mathrm{O} 2 & 0.9(2) \\ \mathrm{C} 1-\mathrm{C} 2-\mathrm{C} 3-\mathrm{C} 1^{\mathrm{i}} & 3.3(2) \\ \mathrm{Br} 1-\mathrm{C} 2-\mathrm{C} 3-\mathrm{C} 1^{\mathrm{i}} & -179.29(9) \\ \mathrm{O} 3-\mathrm{C} 4-\mathrm{C} 5-\mathrm{C} 6 & -178.51(15) \\ \mathrm{C} 6{ }^{\mathrm{ii}}-\mathrm{C} 4-\mathrm{C} 5-\mathrm{C} 6 & 0.5(2) \\ \mathrm{O} 3-\mathrm{C} 4-\mathrm{C} 5-\mathrm{Br} 2 & 2.7(2)\end{array}$

$\mathrm{O} 5-\mathrm{C} 8-\mathrm{H} 8 \mathrm{~A}$

$\mathrm{C} 7-\mathrm{C} 8-\mathrm{H} 8 \mathrm{~A}$

$\mathrm{O} 5-\mathrm{C} 8-\mathrm{H} 8 \mathrm{~B}$

$\mathrm{C} 7-\mathrm{C} 8-\mathrm{H} 8 \mathrm{~B}$

$\mathrm{H} 8 \mathrm{~A}-\mathrm{C} 8-\mathrm{H} 8 \mathrm{~B}$

O5- C9- 10

O5- $\mathrm{C} 9-\mathrm{H} 9 \mathrm{~A}$

$\mathrm{C} 10-\mathrm{C} 9-\mathrm{H} 9 \mathrm{~A}$

$\mathrm{O} 5-\mathrm{C} 9-\mathrm{H} 9 \mathrm{~B}$

$\mathrm{C} 10-\mathrm{C} 9-\mathrm{H} 9 \mathrm{~B}$

H9A $-\mathrm{C} 9-\mathrm{H} 9 \mathrm{~B}$

$\mathrm{N} 1-\mathrm{C} 10-\mathrm{C} 9$

$\mathrm{N} 1-\mathrm{C} 10-\mathrm{H} 10 \mathrm{~A}$

C9- $\mathrm{C} 10-\mathrm{H} 10 \mathrm{~A}$

$\mathrm{N} 1-\mathrm{C} 10-\mathrm{H} 10 \mathrm{~B}$

C9- $\mathrm{C} 10-\mathrm{H} 10 \mathrm{~B}$

$\mathrm{H} 10 \mathrm{~A}-\mathrm{C} 10-\mathrm{H} 10 \mathrm{~B}$

$\mathrm{C} 6$ ii- $44-\mathrm{C} 5-\mathrm{Br} 2$

$\mathrm{C} 4-\mathrm{C} 5-\mathrm{C} 6-\mathrm{O} 4$

$\mathrm{Br} 2-\mathrm{C} 5-\mathrm{C} 6-\mathrm{O} 4$

$\mathrm{C} 4-\mathrm{C} 5-\mathrm{C} 6-\mathrm{C} 4^{\mathrm{ii}}$

$\mathrm{Br} 2-\mathrm{C} 5-\mathrm{C} 6-\mathrm{C} 4^{\mathrm{ii}}$

$\mathrm{C} 10-\mathrm{N} 1-\mathrm{C} 7-\mathrm{C} 8$

$\mathrm{C} 9-\mathrm{O} 5-\mathrm{C} 8-\mathrm{C} 7$

$\mathrm{N} 1-\mathrm{C} 7-\mathrm{C} 8-\mathrm{O} 5$

$\mathrm{C} 8-\mathrm{O} 5-\mathrm{C} 9-\mathrm{C} 10$

$\mathrm{C} 7-\mathrm{N} 1-\mathrm{C} 10-\mathrm{C} 9$

$\mathrm{O} 5-\mathrm{C} 9-\mathrm{C} 10-\mathrm{N} 1$
109.5

109.5

109.5

109.5

108.1

111.18 (13)

109.4

109.4

109.4

109.4

108.0

108.84 (13)

109.9

109.9

109.9

109.9

108.3

$-178.27(10)$

$-178.95(14)$

$-0.2(2)$

$-0.5(2)$

$178.25(10)$

$-55.03(17)$

$-62.46(17)$

58.77 (17)

62.31 (18)

54.35 (17)

$-57.76(19)$

Symmetry codes: (i) $-x+2,-y+2,-z+1$; (ii) $-x,-y+1,-z$.

Hydrogen-bond geometry $\left(\AA,{ }^{\circ}\right)$

\begin{tabular}{lllll}
\hline$D-\mathrm{H} \cdots A$ & $D-\mathrm{H}$ & $\mathrm{H} \cdots A$ & $D \cdots A$ & $D-\mathrm{H} \cdots A$ \\
\hline $\mathrm{N} 1-\mathrm{H} 1 A \cdots \mathrm{O} 4$ & $0.89(3)$ & $2.02(3)$ & $2.890(2)$ & $167(2)$ \\
$\mathrm{N} 1-\mathrm{H} 1 B \cdots \mathrm{O} 1^{\mathrm{iii}}$ & $0.86(3)$ & $2.16(3)$ & $2.938(2)$ & $150(2)$ \\
$\mathrm{N} 1-\mathrm{H} 1 B \cdots \mathrm{O} 2^{\text {iv }}$ & $0.86(3)$ & $2.28(3)$ & $2.964(2)$ & $136(2)$ \\
$\mathrm{O} 2-\mathrm{H} 2 \cdots \mathrm{O} 4$ & $0.82(3)$ & $1.79(4)$ & $2.5224(16)$ & $148(5)$ \\
$\mathrm{O} 2-\mathrm{H} 2 \cdots \mathrm{O} 3^{\mathrm{ii}}$ & $0.82(3)$ & $2.55(4)$ & $3.0678(18)$ & $122(4)$ \\
$\mathrm{O} 4-\mathrm{H} 4 \cdots \mathrm{O} 2$ & $0.82(3)$ & $1.80(4)$ & $2.5224(16)$ & $147(4)$ \\
$\mathrm{C} 7-\mathrm{H} 7 A \cdots \mathrm{O} 4^{\text {iv }}$ & 0.99 & 2.55 & $3.402(2)$ & 145 \\
$\mathrm{C} 10-\mathrm{H} 10 B \cdots B^{\mathrm{n}}{ }^{\mathrm{v}}$ & 0.99 & 2.91 & $3.8946(17)$ & 174 \\
\hline
\end{tabular}

Symmetry codes: (ii) $-x,-y+1,-z$; (iii) $x-1, y-1, z$; (iv) $-x+1,-y+1,-z+1$; (v) $-x,-y,-z$. 